\title{
Circumferentially-Symmetric Finite Eigenstrains in Incompressible Isotropic Nonlinear Elastic Wedges
}

\author{
Ashkan Golgoon ${ }^{\mathrm{a}}$, Souhayl Sadik ${ }^{\mathrm{a}}$, Arash Yavari ${ }^{\mathrm{a}, \mathrm{b}, *}$ \\ ${ }^{a}$ School of Civil and Environmental Engineering, Georgia Institute of Technology, Atlanta, GA 30332, USA \\ ${ }^{b}$ The George W. Woodruff School of Mechanical Engineering, Georgia Institute of Technology, Atlanta, GA 30332, USA
}

\begin{abstract}
Eigenstrains are created as a result of anelastic effects such as defects, temperature changes, bulk growth, etc., and strongly affect the overall response of solids. In this paper, we study the residual stress and deformation fields of an incompressible, isotropic, infinite wedge due to a circumferentially-symmetric distribution of finite eigenstrains. In particular, we establish explicit exact solutions for the residual stresses and deformation of a neoHookean wedge containing a symmetric inclusion with finite radial and circumferential eigenstrains. In addition, we numerically solve for the residual stress field of a neo-Hookean wedge induced by a symmetric Mooney-Rivlin inhomogeneity with finite eigenstrains.
\end{abstract}

Keywords: Finite eigenstrains; residual stresses; nonlinear elasticity; elastic wedge.

\section{Introduction}

The governing equations of nonlinear elasticity are formidably complicated and are amenable to analytic solutions only for very few problems. Semiinverse methods have been particularly useful for obtaining exact solutions for nonlinear elasticity problems. One problem that has attracted several researchers in the last few decades is that of an infinite wedge made of a nonlinear elastic solid (either compressible or incompressible) under various boundary conditions and in the absence of body forces.

Tao and Rajagopal [1] studied the inhomogeneous deformation of a wedge made of a Blatz-Ko material. They assumed a specific form of deformations in which radial planes in the reference configuration remain radial planes after deformation. They found the only possible inhomogeneous solution, which turned out to be asymmetric with respect to the bisecting plane of the wedge. This specific class of deformations was further studied in the literature to find the inhomogeneous deformations in wedges and cones. Fu et al. [2] explored circumferentially-symmetric finite deformations of a wedge made of an incompressible Mooney-Rivlin material. To solve the problem, they specified the translation and rotation of the lateral faces of the wedge. They proved that the deformation is homogeneous when the pressure field associated with the incompressibility condition is uniform. For the inhomogeneous solutions, they were able to reduce the governing equations to a convenient form that allowed for a plane-phase analysis. They observed that for certain wedge angles, the deformation of the wedge is not radially-unidirectional, i.e., some parts of the wedge radially stretch, while others contract. Rajagopal and Carroll [3] assumed inhomogeneous circumferentially-symmetric finite deformations of a wedge made of an isotropic material. Using the displacement lateral boundary conditions and by applying the required tractions on the circular boundary, they obtained, when the material is compressible, a necessary condition that the energy function needs to satisfy for the assumed inhomogeneous deformation to be possible. For incompressible materials, they showed that such an inhomogeneous deformation is possible if the pressure field has a logarithmic singularity at the origin. Rajagopal and Tao [4] studied inhomogeneous circumferentially-symmetric finite deformations of a wedge made of an incompressible power law material. They showed that a "boundary layer solution", i.e., one that is homogeneous in the interior of the wedge but is inhomogeneous close to the boundary, is possible with a bounded pressure field. However, they showed that inhomogeneous solutions are possible only if the pressure field develops a logarithmic singularity at the apex of the wedge. Walton and Wilber [5] investigated the deformations of a neoHookean elastic wedge considering the aforementioned class of deformations. They observed that homogeneous non-unidirectional deformations are possible in every incompressible, isotropic, hyperelastic material. Assuming a more general class of deformations, where some restrictions on the form of the de-

${ }^{*}$ Corresponding author, e-mail: arash.yavari@ce.gatech.edu 
formation were relaxed, they showed that there exist no additional solutions. Walton [6] studied the stability of this class of deformations under small amplitude vibrational perturbations of the lateral faces of a wedge. He found that even to the first order in an asymptotic expansion of the amplitude of the lateral sides of the wedge, the vibrations cannot remain planar; rather out-of-plane vibrational modes must be excited in the interior of the wedge.

In continuum mechanics a strain is some measure of deformation that gives the length of an infinitesimal line element assuming that the length of this line element is known in some other (reference) configuration. A stress is usually defined to be an areal density of force. Given a pair of thermodynamicallyconjugate stress and strain, e.g. the first PiolaKirchhoff stress and the deformation gradient $(\mathbf{P}, \mathbf{F})$ or the second Piola-Kirchhoff stress and the right Cauchy-Green strain $(\mathbf{S}, \mathbf{C})$, locally a non-zero strain does not correspond to a non-zero stress. That part of strain that locally is related to the corresponding stress is called elastic strain. The remaining part is usually referred to as eigenstrain or pre-strain. The term eigenstrain was first used by Mura [7]. Other terms have been used in the literature for the same concept, e.g. initial strain [8], inherent strain [9], and transformation strain [10] (see [11] for a more detailed discussion). In a homogeneous body by an inclusion we mean a region with a distribution of eigenstrains. When the region with eignstrains and the matrix are made of different materials instead of inclusion we use inhomogeneity with eigenstrain.

In the setting of linear elasticity [10] computed the stress field of an ellipsoidal inclusion with uniform (infinitesimal) eigenstrains in an infinite isotropic solid. There have been a few 2D extensions of Eshleby's problem to finite elasticity for harmonic materials [12-16]. The classical shrink-fit problem of nonlinear elasticity [17] is the nonlinear analogue of an inclusion with pure dilatational eigenstrains. The problem of finite eigenstrains in 3D nonlinear elasticity was analytically studied by [18]. They calculated the residual stress fields induced by finite radial and circumferential eigenstrains for the case of spherical balls and (finite and infinite) circular cylindrical bars made of arbitrary incompressible and isotropic solids. The problem of finite shear eigenstrains and the twistfit problem were investigated recently by [19].

To our best knowledge, finite eigenstrains in the framework of nonlinear elasticity have not been studied in any geometry other than spherical and cylindrical. In this paper, we consider an infinite wedge made of an incompressible and isotropic solid and assume that it has a circumferentially-symmetric distribution of finite radial and circumferential eigenstrains. We derive the governing equilibrium equations of the wedge and using a semi-inverse method and assuming a specific class of deformations find the stresses that are induced by finite radial and circumferential eigenstrains. In particular, we solve for the stress field of both neo-Hookean and Mooney-Rivlin wedges with a symmetric inclusion or inhomogeneity with eigenstrains.

This paper is organized as follows. In section 2, we tersely review some basic concepts of geometric anelasticity. In section 3, we discuss the material manifold of a wedge with a circumferentially-symmetric distribution of finite eigenstrains and find the governing equations for an incompressible, isotropic wedge. In sections 3.1 and 3.2, we solve the problems of an inclusion and a Mooney-Rivlin inhomogeneity with uniform eigenstrains in a neo-Hookean wedge. In section 3.3, we find the impotent (stress-free) circumferentially-symmetric finite eigenstrain distributions. In section 4, we conclude the paper with some remarks.

\section{Elements of Geometric Anelasticity}

In this section, we briefly review some fundamental elements of the geometric theory of nonlinear elasticity and anelasticity. For more detailed discussions, see $[20,21]$.

Kinematics.. A body $B$ is assumed to be identified with a Riemannian manifold $(\mathcal{B}, \boldsymbol{G})$. A configuration of $\mathcal{B}$ is a smooth embedding $\varphi: \mathcal{B} \rightarrow \mathcal{S}$, where $(\mathcal{S}, \boldsymbol{g})$ is the Euclidean ambient space. We denote by $\nabla^{\boldsymbol{G}}$ and $\nabla^{g}$ the Levi-Civita connections associated with the Riemannian manifolds $(\mathcal{B}, \boldsymbol{G})$ and $(\mathcal{S}, \boldsymbol{g})$, respectively. The set of all configurations of $\mathcal{B}$ is denoted by $C$. A motion of $\mathcal{B}$ is a curve $\mathbb{R}^{+} \rightarrow \varphi_{t} \in C$ such that $\varphi_{t}$ assigns a spatial point $x=\varphi_{t}(X)=\varphi(X, t) \in \mathcal{S}$ to every material point $X \in \mathcal{B}$ at a time $t$. The deformation gradient $\boldsymbol{F}$ is the derivative map of $\varphi_{t}$ defined as

$$
\boldsymbol{F}(X, t)=d \varphi_{t}(X): T_{X} \mathcal{B} \rightarrow T_{\varphi_{t}(X)} \mathcal{S} .
$$

The adjoint of $\boldsymbol{F}$ is defined by

$$
\begin{aligned}
\boldsymbol{F}^{\top}(X, t) & : T_{\varphi_{t}(X)} \mathcal{S} \rightarrow T_{X} \mathcal{B}, \\
& \boldsymbol{g}(\boldsymbol{F} \boldsymbol{V}, \mathbf{v})=\boldsymbol{G}\left(\boldsymbol{V}, \boldsymbol{F}^{\top} \mathbf{v}\right), \\
& \forall \boldsymbol{V} \in T_{X} \mathcal{B}, \mathbf{v} \in T_{\varphi_{t}(X)} \mathcal{S} .
\end{aligned}
$$

The right Cauchy-Green deformation tensor is defined as

$$
\boldsymbol{C}(X, t)=\boldsymbol{F}^{\top}(X, t) \boldsymbol{F}(X, t): T_{X} \mathcal{B} \rightarrow T_{X} \mathcal{B} .
$$

In the coordinate charts $\left\{X^{A}\right\}$ and $\left\{x^{a}\right\}$ for $\mathcal{B}$ and $\mathcal{S}$, respectively, in components, $\boldsymbol{C}$ can be written as: $C^{A}{ }_{B}=G^{A L} F^{a}{ }_{L} F^{b}{ }_{B} g_{a b}$. The Jacobian of the motion $J$ relates the material and spatial Riemannian volume elements $d V(X, \boldsymbol{G})$ and $d v(x, \boldsymbol{g})$ by $d v=J d V$ and is given by

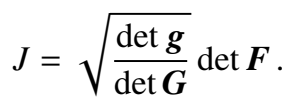


Constitutive equations.. In this paper we restrict our calculations to incompressible isotropic hyperelastic solids. That is, there exists an energy function $W$ that depends only on the first two principal invariants of $\mathbf{C}: I_{1}=\operatorname{tr} \boldsymbol{C}$ and $I_{2}=\frac{1}{2}\left(\operatorname{tr}(\boldsymbol{C})^{2}-\operatorname{tr}\left(\boldsymbol{C}^{2}\right)\right)$, i.e., $W=W\left(X, I_{1}, I_{2}\right)$, such that the Cauchy stress tensor is given in components by [22]

$$
\begin{array}{r}
\sigma^{a b}=2 F^{a}{ }_{A} F^{b}{ }_{B}\left[\left(W_{I_{1}}+I_{1} W_{I_{2}}\right) G^{A B}\right. \\
\left.-W_{I_{2}} C^{A B}\right] \\
-p g^{a b},
\end{array}
$$

where $W_{I_{1}}:=\frac{\partial W}{\partial I_{1}}, W_{I_{2}}:=\frac{\partial W}{\partial I_{2}}$, and $p$ is the Lagrange multiplier associated with the internal incompressibility constraint $J=1$.

Equilibrium equations.. In terms of the Cauchy stress tensor, the localized balance of linear momentum of a body in static equilibrium and in the absence of body forces reads

$$
\operatorname{div} \boldsymbol{\sigma}=\mathbf{0},
$$

where div denotes the spatial divergence operator. In components, the spatial divergence operator reads

$$
(\operatorname{div} \sigma)^{a}=\sigma^{a b}{ }_{b b}=\frac{\partial \sigma^{a b}}{\partial x^{b}}+\sigma^{a c} \gamma_{c b}^{b}+\sigma^{c b} \gamma_{c b}^{a},
$$

where $\gamma_{b c}^{a}$ is the Christoffel symbol of the LeviCivita connection $\nabla^{g}$ in the local chart $\left\{x^{a}\right\}$, defined as $\nabla^{g}{ }_{\partial_{b}} \partial_{c}=\gamma_{b c}^{a} \partial_{a}$, (similarly, for the material manifold $\nabla^{\boldsymbol{G}_{\partial_{B}} \partial_{C}}=\Gamma_{B C}^{A} \partial_{A}$ ).

The Riemannian material manifold.. In geometric anelasticity one starts with a stress-free body $\mathcal{B}$ without eigenstrains sitting in the Euclidean space with metric $\mathbf{G}_{0}$. This means that the body free of eigenstrains is a Riemannian manifold $\left(\mathcal{B}, \mathbf{G}_{0}\right)$. The effect of an eigenstrain distribution is to locally transform a line element $d \mathbf{X}_{0}$ to $d \mathbf{X}=\mathbf{K} d \mathbf{X}_{0}$, where $\mathbf{K}$ explicitly depends on the distribution of eigenstrains. Note that

$$
\mathbf{G}_{0}\left(d \mathbf{X}_{0}, d \mathbf{X}_{0}\right)=\mathbf{G}(d \mathbf{X}, d \mathbf{X}),
$$

where $\mathbf{G}=\mathbf{K}_{*} \mathbf{G}_{0}$ is the push-forward of $\mathbf{G}_{0}$ by $\mathbf{K}$. In the manifold $(\mathcal{B}, \mathbf{G})$, the body with the distributed eigenstrains is stress-free because the distances are set to be those of the hypothetically relaxed body. Note that in components, $G_{A B}=K^{\alpha}{ }_{A} K^{\beta}{ }_{B}\left(G_{0}\right)_{\alpha \beta}$, where the coordinate charts $\left\{\bar{X}^{\alpha}\right\}$ and $\left\{X^{A}\right\}$ in the initial and distorted reference configurations, respectively, are assumed.

In the geometric formulation of anelasticity, all the anelastic effects are buried into the material manifold. In other words, if one succeeds in building a material manifold (where the body is stress-free by construction) then the anelasticity problem is transformed into a classical nonlinear elasticity problem as long as the non-trivial geometry of the material manifold is taken into account properly. In our formulation of nonlinear anelasticity kinematics and the governing equations have forms identical to those of the classical nonlinear elasticity; nonlinear elasticity is a special case in this formulation in which the material manifold is $\mathrm{Eu}-$ clidean. Certain questions, e.g. finding the stress-free finite eigenstrain distributions, are formulated quite naturally in the geometric framework as we will explain in this paper.

\section{An infinite incompressible isotropic wedge with finite circumferentially symmetric eigenstrains}

In this section we consider an infinitely long wedge of radius $R_{o}$ and angle $2 \Theta_{o}$ (see Figure 1). Let $(\bar{R}, \bar{\Theta}, \bar{Z})$ be the cylindrical coordinates for which $\bar{R} \geq 0,-\Theta_{o} \leq \bar{\Theta} \leq \Theta_{o}$, and $\bar{Z} \in \mathbb{R}$ such that the axis of the wedge corresponds to $\bar{R}=0$. In the cylindrical coordinates $(\bar{R}, \bar{\Theta}, \bar{Z})$, the material metric for the eigenstrain-free configuration reads

$$
\boldsymbol{G}_{0}=\left(\begin{array}{ccc}
1 & 0 & 0 \\
0 & \bar{R}^{2} & 0 \\
0 & 0 & 1
\end{array}\right)
$$

We assume a circumferentially-symmetric eigenstrain (pre-strain) distribution in the wedge. With respect to the initial reference configuration and using the cylindrical coordinates $(R, \Theta, Z)$ for the material manifold $\mathbf{K}$ is assumed to have the following representation

$$
\boldsymbol{K}=\left(\begin{array}{ccc}
e^{\omega_{R}(\Theta)} & 0 & 0 \\
0 & e^{\omega_{\Theta}(\Theta)} & 0 \\
0 & 0 & 1
\end{array}\right),
$$

where $\omega_{R}(\Theta)$ and $\omega_{\Theta}(\Theta)$ are arbitrary functions that describe the radial and circumferential eigenstrain distributions in the wedge. Now the material metric $\mathbf{G}=\mathbf{K}_{*} \mathbf{G}_{0}$ will have the following representation in the cylindrical coordinates $(R, \Theta, Z)$ :

$$
\boldsymbol{G}=\left(\begin{array}{ccc}
e^{2 \omega_{R}(\Theta)} & 0 & 0 \\
0 & R^{2} e^{2 \omega_{\Theta}(\Theta)} & 0 \\
0 & 0 & 1
\end{array}\right) .
$$

This is the metric that was introduced by Yavari and Goriely [18]. ${ }^{1}$

\footnotetext{
${ }^{1}$ Similar constructions using non-trivial material geometries have been introduced in thermoelasticity, growth mechanics, and the mechanics of distributed defects [21, 23-29].
} 


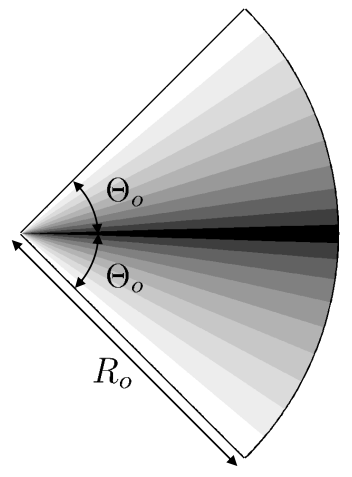

Figure 1: A wedge with a finite circumferentially-symmetric eigenstrain distribution.

We endow the ambient space with the flat Euclidean metric, which in cylindrical coordinates $(r, \theta, z)$ reads

$$
\boldsymbol{g}=\left(\begin{array}{ccc}
1 & 0 & 0 \\
0 & r^{2} & 0 \\
0 & 0 & 1
\end{array}\right)
$$

Let us consider the class of deformations for which radial surfaces $\Theta=$ constant in the reference configuration remain planar and are mapped to radial surfaces in the current configuration. That is, we assume an embedding of the material manifold into the ambient space with the following form

$$
r=k(R, \Theta), \quad \theta=h(\Theta), \quad z=Z .
$$

Therefore, the deformation gradient reads

$$
\boldsymbol{F}=\left(\begin{array}{ccc}
\frac{\partial k}{\partial R} & \frac{\partial k}{\partial \Theta} & 0 \\
0 & \frac{d h}{d \Theta} & 0 \\
0 & 0 & 1
\end{array}\right)
$$

Assuming incompressibility $J=\sqrt{\frac{\operatorname{det} g}{\operatorname{det} G}} \operatorname{det} \boldsymbol{F}=1$, we find

$$
h^{\prime}(\Theta)\left[k^{2}(R, \Theta)-k^{2}(0, \Theta)\right]=R^{2} e^{\omega_{R}(\Theta)+\omega_{\Theta}(\Theta)} .
$$

Eliminating the rigid body translation by setting $r(0, \Theta)=0$, we find that

$$
r=k(R, \Theta)=R \zeta(\Theta),
$$

where

$$
\zeta^{2}(\Theta)=\frac{e^{\omega_{R}(\Theta)+\omega_{\Theta}(\Theta)}}{h^{\prime}(\Theta)} .
$$

This means that for an incompressible wedge within the class of deformations (3.5), and given the radial and circumferential eigenstrain distributions, the kinematics is fully determined after solving for the unknown function $\zeta=\zeta(\Theta)$. The right Cauchy-Green deformation tensor is written as

$$
\boldsymbol{C}=\left(\begin{array}{ccc}
e^{-2 \omega_{R}(\Theta)} \zeta^{2}(\Theta) & R e^{-2 \omega_{R}(\Theta)} \zeta(\Theta) \zeta^{\prime}(\Theta) & 0 \\
\frac{e^{-2 \omega_{\Theta}(\Theta)}}{R} \zeta(\Theta) \zeta^{\prime}(\Theta) & \frac{e^{2 \omega_{N}(\Theta)}}{\zeta^{2}(\Theta)}+\zeta^{\prime}(\Theta)^{2} e^{-2 \omega_{\Theta}(\Theta)} & 0 \\
0 & 0 & 1
\end{array}\right) .
$$

The invariants of $\boldsymbol{C}$ are

$$
\begin{aligned}
I_{1} & =\operatorname{tr}(\boldsymbol{C})=1+\zeta(\Theta)^{2} e^{-2 \omega_{R}(\Theta)}+\zeta^{\prime}(\Theta)^{2} e^{-2 \omega_{\Theta}(\Theta)} \\
& +\frac{e^{2 \omega_{R}(\Theta)}}{\zeta(\Theta)^{2}} \\
I_{2} & =\frac{1}{2}\left(\operatorname{tr}\left(\boldsymbol{C}^{2}\right)-\operatorname{tr}(\boldsymbol{C})^{2}\right)=1+\zeta(\Theta)^{2} e^{-2 \omega_{R}(\Theta)} \\
& +\zeta^{\prime}(\Theta)^{2} e^{-2 \omega_{\Theta}(\Theta)}+\frac{e^{2 \omega_{R}(\Theta)}}{\zeta(\Theta)^{2}} \\
I_{3} & =\operatorname{det}(\boldsymbol{C})=1 .
\end{aligned}
$$

Note that $I_{1}=I_{2}$ depends only on $\Theta$.

We assume that the wedge is made of an incompressible isotropic radially-homogenous material, i.e., the strain energy function has the form $W=$ $W\left(\Theta, I_{1}, I_{2}\right)$. Following (2.5), for the class of deformations (3.5), the non-zero components of the Cauchy stress tensor read

$$
\begin{aligned}
\sigma^{r r}= & 2\left(W_{I_{1}}+W_{I_{2}}\right)\left(e^{-2 \omega_{\Theta}(\Theta)} \zeta^{\prime}(\Theta)^{2}+\zeta(\Theta)^{2} e^{-2 \omega_{R}(\Theta)}\right) \\
& -p+2 W_{I_{2}},
\end{aligned}
$$

$\sigma^{r \theta}=\frac{2 \zeta^{\prime}(\Theta)}{R \zeta(\Theta)^{2}}\left(W_{I_{1}}+W_{I_{2}}\right) e^{\omega_{R}(\Theta)-\omega_{\Theta}(\Theta)}$,

$$
\begin{aligned}
\sigma^{\theta \theta}= & \frac{1}{R^{2} \zeta(\Theta)^{4}}\left(2 e^{2 \omega_{R}(\Theta)}\left(W_{I_{1}}+W_{I_{2}}\right)\right. \\
& \left.-\zeta(\Theta)^{2}\left(p-2 W_{I_{2}}\right)\right)
\end{aligned}
$$

$$
\begin{aligned}
\sigma^{z z}= & 2 W_{I_{2}}\left(e^{-2 \omega_{\Theta}(\Theta)} \zeta^{\prime}(\Theta)^{2}+\zeta(\Theta)^{2} e^{-2 \omega_{R}(\Theta)}+\frac{e^{2 \omega_{R}(\Theta)}}{\zeta(\Theta)^{2}}\right) \\
& -p+2 W_{I_{1}} .
\end{aligned}
$$

The physical components of the Cauchy stress, i.e., $\hat{\sigma}^{a b}=\sigma^{a b} \sqrt{g_{a a} g_{b b}}$ (no summation) [30] read

$$
\hat{\sigma}^{r r}=\sigma^{r r}, \quad \hat{\sigma}^{r \theta}=R \zeta(\Theta) \sigma^{r \theta}, \quad \begin{aligned}
\hat{\sigma}^{\theta \theta} & =R^{2} \zeta^{2}(\Theta) \sigma^{\theta \theta}, \\
\hat{\sigma}^{z z} & =\sigma^{z z} .
\end{aligned}
$$

The first Piola-Kirchhoff stress tensor $P^{a A}=$ $J\left(F^{-1}\right)_{b}^{A} \sigma^{a b}$ has the following non-zero components

$$
\begin{aligned}
P^{r R}=\frac{e^{-2 \omega_{R}(\Theta)}}{\zeta(\Theta)} & \left(2 \zeta(\Theta)^{2}\left(W_{I_{1}}+W_{I_{2}}\right)\right. \\
- & \left.e^{2 \omega_{R}(\Theta)}\left(p-2 W_{I_{2}}\right)\right),
\end{aligned}
$$

$$
\begin{aligned}
& P^{r \Theta}=\frac{2 e^{-2 \omega_{\Theta}(\Theta)}}{R} \zeta^{\prime}(\Theta)\left(W_{I_{1}}+W_{I_{2}}\right), \\
& P^{\theta R}=\frac{e^{-\omega_{\Theta}(\Theta)-\omega_{R}(\Theta)} \zeta^{\prime}(\Theta)}{R \zeta(\Theta)}\left(p-2 W_{I_{2}}\right),
\end{aligned}
$$




$$
\begin{aligned}
P^{\Theta \Theta}=\frac{e^{-\omega_{\Theta}(\Theta)-\omega_{R}(\Theta)}}{R^{2} \zeta(\Theta)^{2}} & \left(2 e^{2 \omega_{R}(\Theta)}\left(W_{I_{1}}+W_{I_{2}}\right)\right. \\
& \left.-\zeta(\Theta)^{2}\left(p-2 W_{I_{2}}\right)\right),
\end{aligned}
$$

$$
\begin{aligned}
P^{z Z}= & 2 W_{I_{2}}\left(e^{-2 \omega_{\Theta}(\Theta)} \zeta^{\prime}(\Theta)^{2}+\zeta(\Theta)^{2} e^{-2 \omega_{R}(\Theta)}+\frac{e^{2 \omega_{R}(\Theta)}}{\zeta(\Theta)^{2}}\right) \\
& -p+2 W_{I_{1}} .
\end{aligned}
$$

In the absence of body forces, the non-trivial equilibrium equations are $\sigma^{r b}{ }_{b b}=0$ and $\sigma^{\theta b}{ }_{b b}=0$ (the axial equilibrium equation implies that $p=p(R, \Theta)$ ). Note that, following (3.5), (3.8), and (3.9), we have

$$
\begin{aligned}
\frac{\partial}{\partial r} & =\frac{1}{\zeta(\Theta)} \frac{\partial}{\partial R}, \\
\frac{\partial}{\partial \theta} & =\frac{\zeta^{2}(\Theta)}{e^{\omega_{R}(\Theta)+\omega_{\Theta}(\Theta)}}\left(\frac{\partial}{\partial \Theta}-\frac{R \zeta^{\prime}(\Theta)}{\zeta(\Theta)} \frac{\partial}{\partial R}\right) .
\end{aligned}
$$

Therefore, the non-trivial equilibrium equations read

$$
\begin{gathered}
2 \zeta e^{-2 \omega_{\Theta}}\left[\zeta^{\prime \prime}\left(W_{I_{1}}+W_{I_{2}}\right)+\zeta^{\prime}\left(W_{I_{1}}+W_{I_{2}}\right)\left(\omega_{R}^{\prime}-\omega_{\Theta}^{\prime}\right)\right. \\
\left.+\zeta^{\prime}\left(W_{I_{1}}^{\prime}+W_{I_{2}}^{\prime}\right)\right]+2\left(W_{I_{1}}+W_{I_{2}}\right)\left[\zeta^{2} e^{-2 \omega_{R}}-\frac{e^{2 \omega_{R}}}{\zeta^{2}}\right] \\
-R \frac{\partial p}{\partial R}=0,
\end{gathered}
$$

$$
\begin{aligned}
& R \zeta \zeta^{\prime} \frac{\partial p}{\partial R}-\zeta^{2} \frac{\partial p}{\partial \Theta}+2 e^{2 \omega_{R}}\left(W_{I_{1}}^{\prime}+W_{I_{2}}^{\prime}\right) \\
& +4 e^{2 \omega_{R}} \omega_{R}^{\prime}\left(W_{I_{1}}+W_{I_{2}}\right)+2 \zeta^{2} W_{I_{2}}^{\prime}=0,
\end{aligned}
$$

where by using the chain rule, one can write

$$
\begin{aligned}
& W_{I_{1}}^{\prime}(\Theta)=\frac{\partial W_{I_{1}}}{\partial I_{1}} \frac{\partial I_{1}}{\partial \Theta}+\frac{\partial W_{I_{1}}}{\partial I_{2}} \frac{\partial I_{2}}{\partial \Theta}+\frac{\partial W_{I_{1}}}{\partial \Theta}, \\
& W_{I_{2}}^{\prime}(\Theta)=\frac{\partial W_{I_{2}}}{\partial I_{1}} \frac{\partial I_{1}}{\partial \Theta}+\frac{\partial W_{I_{2}}}{\partial I_{2}} \frac{\partial I_{2}}{\partial \Theta}+\frac{\partial W_{I_{2}}}{\partial \Theta} .
\end{aligned}
$$

It follows from (3.26a) that

$$
p(R, \Theta)=f(\Theta) \ln R+\Phi(\Theta),
$$

where

$$
\begin{aligned}
f(\Theta)= & 2 \zeta e^{-2 \omega_{\Theta}}\left[\zeta^{\prime \prime}\left(W_{I_{1}}+W_{I_{2}}\right)+\right. \\
\left.\zeta^{\prime}\left(W_{I_{1}}+W_{I_{2}}\right)\left(\omega_{R}^{\prime}-\omega_{\Theta}^{\prime}\right)+\zeta^{\prime}\left(W_{I_{1}}^{\prime}+W_{I_{2}}^{\prime}\right)\right] & \\
& +2\left(W_{I_{1}}+W_{I_{2}}\right)\left[\zeta^{2} e^{-2 \omega_{R}}-\frac{e^{2 \omega_{R}}}{\zeta^{2}}\right], \quad
\end{aligned}
$$

and $\Phi(\Theta)$ is an arbitrary function of $\Theta$ to be determined. Substituting the pressure field into (3.26b) yields

$$
\begin{array}{r}
\zeta \zeta^{\prime} f-\zeta^{2} \Phi^{\prime}+2 e^{2 \omega_{R}}\left(W_{I_{1}}^{\prime}+W_{I_{2}}^{\prime}\right)+4 e^{2 \omega \omega_{R}} \omega_{R}^{\prime}\left(W_{I_{1}}+W_{I_{2}}\right) \\
+2 \zeta^{2} W_{I_{2}}^{\prime}-\zeta^{2} f^{\prime} \ln R=0 .
\end{array}
$$

Note that (3.30) must hold for any $R$ and $\zeta(\Theta) \neq 0$. Therefore, $f$ is constant, i.e., $f(\Theta)=f_{o}$ and hence

$$
p(R, \Theta)=f_{o} \ln R+\Phi(\Theta) .
$$

Therefore, the equilibrium equation (3.30) is reduced to the following ODE

$$
\begin{aligned}
\zeta \zeta^{\prime} f_{o}- & \zeta^{2} \Phi^{\prime}+4 e^{2 \omega_{R}} \omega_{R}^{\prime}\left(W_{I_{1}}+W_{I_{2}}\right) \\
& +2 e^{2 \omega_{R}} W_{I_{1}}^{\prime}+2\left[e^{2 \omega_{R}}+\zeta^{2}\right] W_{I_{2}}^{\prime}=0 .
\end{aligned}
$$

One then obtains $\Phi^{\prime}$ as

$$
\begin{aligned}
\Phi^{\prime}(\Theta)= & f_{o} \frac{\zeta^{\prime}}{\zeta}+\frac{2 e^{2 \omega_{R}}}{\zeta^{2}}\left(W_{I_{1}}^{\prime}+W_{I_{2}}^{\prime}\right) \\
& +\frac{4 e^{2 \omega_{R}} \omega_{R}^{\prime}}{\zeta^{2}}\left(W_{I_{1}}+W_{I_{2}}\right)+2 W_{I_{2}}^{\prime} .
\end{aligned}
$$

Equation (3.29) gives us the following nonlinear second-order ODE for $\zeta(\Theta)$.

$$
\begin{array}{r}
2 \zeta e^{-2 \omega_{\Theta}}\left[\zeta^{\prime \prime}\left(W_{I_{1}}+W_{I_{2}}\right)+\zeta^{\prime}\left(W_{I_{1}}+W_{I_{2}}\right)\left(\omega_{R}^{\prime}-\omega_{\Theta}^{\prime}\right)\right. \\
\left.+\zeta^{\prime}\left(W_{I_{1}}^{\prime}+W_{I_{2}}^{\prime}\right)\right]+2\left(W_{I_{1}}+W_{I_{2}}\right)\left[\zeta^{2} e^{-2 \omega_{R}}-\frac{e^{2 \omega_{R}}}{\zeta^{2}}\right] \\
=f_{o} .
\end{array}
$$

In the next section, we will solve for the residual stress field of a neo-Hookean wedge with a symmetric inclusion with uniform eigenstrains.

\subsection{An inclusion with uniform eigenstrains in a neo-}

Hookean wedge with traction-free lateral boundaries

Let us consider the following distribution of eigenstrains in the wedge (see Figure 2)

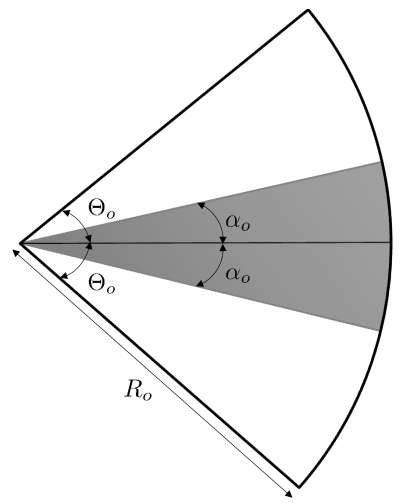

Figure 2: A wedge with uniform eigenstrains in the shaded region.

$$
\begin{aligned}
& \omega_{R}(\Theta)=\left\{\begin{array}{ll}
\omega_{1}, & |\Theta| \leq \alpha_{o} \\
0, & |\Theta|>\alpha_{o}
\end{array},\right. \\
& \omega_{\Theta}(\Theta)=\left\{\begin{array}{ll}
\omega_{2}, & |\Theta| \leq \alpha_{o} \\
0, & |\Theta|>\alpha_{o}
\end{array},\right.
\end{aligned}
$$

where $\omega_{1}$ and $\omega_{2}$ are constants. Let us assume that the wedge is made of an incompressible homogeneous neo-Hookean solid, i.e., $W=W\left(I_{1}\right)=\frac{\mu}{2}\left(I_{1}-3\right)$. Thus, 
$W_{I_{1}}=\frac{\mu}{2}, W_{I_{2}}=0$. Simplifying (3.34), we find the following non-linear second-order ODEs inside and outside the inclusion

$$
\begin{aligned}
\zeta \zeta^{\prime \prime} e^{-2 \omega_{2}}+\zeta^{2} e^{-2 \omega_{1}}-\frac{e^{2 \omega_{1}}}{\zeta^{2}} & =\frac{f_{o}}{\mu}, & |\Theta| \leq \alpha_{o}, \\
\zeta \zeta^{\prime \prime}+\zeta^{2}-\frac{1}{\zeta^{2}} & =\frac{f_{o}}{\mu}, & |\Theta|>\alpha_{o} .
\end{aligned}
$$

Note that in the absence of eigenstrains $\left(\omega_{1}=\omega_{2}=\right.$ 0 ), the above equations reduce to the equation for the deformation of a wedge derived by Fu et al. [2], Rajagopal and Carroll [3], Rajagopal and Tao [4]. We integrate (3.33) for the assumed eigenstrain distribution and find that the pressure field has the following distribution

$$
\begin{aligned}
p(R, \Theta) & =f_{o} \ln R+\Phi(\Theta) \\
& = \begin{cases}f_{o} \ln (R \zeta(\Theta))+p_{i}, & |\Theta| \leq \alpha_{o}, \\
f_{o} \ln (R \zeta(\Theta))+p_{o}, & |\Theta|>\alpha_{o},\end{cases}
\end{aligned}
$$

where $p_{i}$ and $p_{o}$ are constants of integration. We integrate (3.36) once and obtain

$$
\zeta^{\prime}(\Theta)^{2}=\left\{\begin{array}{cc}
c_{i_{1}}+\frac{2 f_{o} e^{2 \omega_{2}}}{\mu} \ln \zeta-\zeta^{2} e^{2\left(\omega_{2}-\omega_{1}\right)} \\
-\frac{e^{2\left(\omega_{1}+\omega_{2}\right)}}{\zeta^{2}}, & |\Theta| \leq \alpha_{o}, \\
c_{O_{1}}+\frac{2 f_{o}}{\mu} \ln \zeta-\zeta^{2} \\
-\frac{1}{\zeta^{2}}, \quad & |\Theta|>\alpha_{o},
\end{array}\right.
$$

where $c_{i_{1}}$ and $c_{o_{1}}$ are constants of integration. In order to solve (3.38) for $\zeta$, we next examine the boundary and continuity conditions.

Boundary conditions.. The traction vector is defined as

$$
\boldsymbol{t}=\langle\boldsymbol{\sigma}, \boldsymbol{n}\rangle_{\boldsymbol{g}}
$$

In components, $t^{a}(x, \boldsymbol{n})=\sigma^{a c} g_{b c} n^{b}$. From (3.39), the continuity of the traction vector on the boundary of the inclusion (or inhomogeneity) implies that both $\sigma^{r \theta}$ and $\sigma^{\theta \theta}$ must be continuous at $\Theta= \pm \alpha_{o}$. Thus, after some simplifications, (3.15) and (3.16) give us

$$
\left.e^{\omega_{1}-\omega_{2}} \zeta^{\prime}(\Theta)\right|_{\Theta=\alpha_{o}^{-}}=\left.\zeta^{\prime}(\Theta)\right|_{\Theta=\alpha_{o}^{+}}
$$

and

$$
\mu\left(e^{2 \omega_{1}}-1\right)=\left(p_{i}-p_{o}\right) \zeta\left( \pm \alpha_{o}\right)^{2} .
$$

Remark 3.1. From (3.41), it is clear that when the eigenstrain distribution is purely circumferential, i.e., $\omega_{1}=0$, one finds that $p_{i}=p_{o}=c$. Hence, the pressure field is continuous at the inclusion boundary and reads $p(R, \Theta)=f_{o} \ln (R \zeta(\Theta))+c$.
Remark 3.2. Note that although the Cauchy traction vector $\boldsymbol{t}(x, \boldsymbol{n})=\langle\boldsymbol{\sigma}, \boldsymbol{n}\rangle_{g}$ is continuous at the inclusion boundary, the first Piola-Kirchhoff traction vector $\boldsymbol{t}_{o}(X, \boldsymbol{N})=\langle\boldsymbol{P}, \boldsymbol{N}\rangle_{\boldsymbol{G}}$ is not. This is due to the fact that $\boldsymbol{t}_{o}$ is defined with respect to the undeformed surface element $d A$ in the reference configuration. Since the material metric is discontinuous at the inclusion boundary, $d A$ is discontinuous as well. However, $\boldsymbol{t}_{o}(X, \boldsymbol{N}) d A=\boldsymbol{t}(x, \boldsymbol{n}) d a$ is continuous. Hence the first Piola-Kirchhoff traction vector must be discontinuous at the inclusion boundary to account for the discontinuity of $d A$ and make $\boldsymbol{t}_{o}(X, N) d A$ continuous. On the other hand, $t$ is continuous because it is defined per unit of deformed area in the current configuration $d a$, which is continuous at the inclusion boundary.

The continuity of the displacement field implies that $\zeta(\Theta)$ and $h(\Theta)$ are both continuous at $\Theta= \pm \alpha_{o}$. For boundary conditions, we can either prescribe the tractions or the resultant forces acting on the boundary of the wedge. Alternatively, we may specify the boundary displacements and then find the required surface tractions. We assume the special case of symmetric boundary conditions with respect to the bisecting plane of the wedge, and then find the boundary tractions required to maintain such a deformation. Note, however, that Tao and Rajagopal [1] showed that for Blatz-Ko (compressible) materials, only asymmetric inhomogeneous solutions are admitted by the equilibrium equations.

Let us assume that the lateral boundaries are traction-free, i.e.

$$
P^{r \Theta}=P^{\theta \Theta}=0, \quad 0 \leq R \leq R_{o}, \quad \Theta= \pm \Theta_{o} .
$$

Imposing (3.42), we find that $p(R, \Theta)$ must be bounded $\left(f_{o}=0\right)$ and

$$
\zeta^{\prime}\left( \pm \Theta_{o}\right)=0, \quad p_{o}=\frac{\mu}{\zeta\left(\Theta_{o}\right)^{2}} .
$$

Furthermore, (3.37) implies that the pressure is equal to $p_{i}$ inside the inclusion and is equal to $p_{o}$ outside the inclusion. Note that due to the symmetry of the problem, $\zeta(\Theta)$ and $h(\Theta)$ must be even and odd, respectively. Thus, since (3.36) implies that $\zeta(\Theta)$ must be at least $C^{2}$ inside the inclusion, we have $\zeta^{\prime}(0)=0$. Hence, we can solve the problem by imposing the above boundary conditions, which in turn specify the required traction distribution on the circular boundary of the wedge. Then, we find the resultant force acting on the circular boundary of the wedge, which is equal to the force that needs to be applied at the apex of the wedge to maintain the equilibrium. The radial material traction per unit undeformed area acting on the circular boundary is calculated using the relation, $t_{o}^{a}=P^{a A} N^{B} G_{B A}$. Thus ${ }^{2}$

$$
t_{o}^{\theta}=\hat{P}^{\theta R}, t_{o}^{r}=\hat{P}^{r R}, R=R_{o},-\Theta_{o}<\Theta<\Theta_{o} .
$$

\footnotetext{
${ }^{2}$ Note that the physical components of the first Piola-Kirchhoff stress tensor are defined as $\hat{P}^{a A}=P^{a A} \sqrt{G_{A A} g_{a a}}$ (no summation).
} 
Therefore, the radial force per unit undeformed area reads $^{3}$

$$
F_{r}=\int t_{o}^{r} d A_{G},
$$

where $d A_{G}=R_{o} e^{\omega_{\Theta}(\Theta)} d \Theta \wedge d Z$ is the Riemannian area element. ${ }^{4}$ Hence, for the infinite cylinder (in the $Z$ direction), the radial force per unit length of the cylinder in the $Z$-direction is written as

$$
F_{r}=\int_{-\Theta_{o}}^{\Theta_{o}}\left(\left.R_{o} e^{\omega_{\Theta}(\Theta)} e^{\omega_{R}(\Theta)} P^{r R}\right|_{\left(R_{o}, \Theta\right)}\right) d \Theta,
$$

which is simplified to read

$$
\begin{array}{r}
F_{r}=2 \mu R_{o}\left[e^{\omega_{2}-\omega_{1}} \int_{0}^{\alpha_{o}}\left(\zeta(\Theta)-\frac{p_{i} e^{2 \omega_{1}}}{\mu \zeta(\Theta)}\right) d \Theta\right. \\
\left.+\int_{\alpha_{o}}^{\Theta_{o}}\left(\zeta(\Theta)-\frac{p_{o}}{\mu \zeta(\Theta)}\right) d \Theta\right] .
\end{array}
$$

Remark 3.3. It is worth mentioning that only if $\zeta(\Theta)=$ constant one can enforce pointwise zero traction boundary conditions on the whole boundary of the wedge for any values of $\omega_{1}$. In this case, we can only have $\Theta_{o}=\alpha_{o}$ and $\zeta=e^{\omega_{1}}$, which in turn gives $h(\Theta)=e^{\omega_{2}-\omega_{1}} \Theta$. Hence, all the stress components vanish point-wise.
Solving (3.38), one obtains $\zeta(\Theta)$ in the upper half region of the wedge as ${ }^{5}$

$$
\zeta(\Theta)=\left\{\begin{array}{c}
e^{\frac{1}{2}\left(\omega_{1}-\omega_{2}\right)}\left[\left(\sqrt{\left(\frac{1}{2} c_{i_{1}} e^{\omega_{1}-\omega_{2}}\right)^{2}-e^{2\left(\omega_{1}+\omega_{2}\right)}}\right)\right. \\
\cos \left(2 e^{\omega_{2}-\omega_{1}} \Theta+c_{i_{2}}\right) \\
\left.+\frac{1}{2} c_{i_{1}} e^{\omega_{1}-\omega_{2}}\right]^{\frac{1}{2}}, \quad 0 \leq \Theta \leq \alpha_{o}, \\
{\left[\left(\sqrt{\frac{1}{4} c_{o_{1}}^{2}-1}\right) \cos \left(2 \Theta+c_{o_{2}}\right)\right.} \\
\left.+\frac{1}{2} c_{o_{1}}\right]^{\frac{1}{2}}, \quad \alpha_{o} \leq \Theta \leq \Theta_{o},
\end{array}\right.
$$

where $c_{i_{2}}$ and $c_{O_{2}}$ are constants.

Equation (3.43), i.e., $\zeta^{\prime}\left(\Theta_{o}\right)=0$, and $\zeta^{\prime}(0)=0$ give us $c_{O_{2}}=k_{1} \pi-2 \Theta_{o}$ and $c_{i_{2}}=k_{2} \pi$, respectively, where $k_{1}, k_{2} \in \mathbb{Z}$. Upon using the continuity of $\zeta(\Theta)$ at $\Theta=\alpha_{o}$ as well as (3.40), we find $c_{i_{1}}$ and $c_{o_{1}}$. They read

$$
\begin{aligned}
c_{i_{1}}= & 2 e^{2 \omega_{2}}\left[1+\left(e^{4 \omega_{1}}-1\right)^{2}\left\{-e^{4 \omega_{1}}\left(e^{4 \omega_{1}}+1\right) \cot \left(2\left(\alpha_{o}-\Theta_{o}\right)\right) \sin \left(4 e^{\omega_{2}-\omega_{1}} \alpha_{o}\right)\right.\right. \\
& +e^{4 \omega_{1}} \csc ^{2}\left(2\left(\alpha_{o}-\Theta_{o}\right)\right) \sin ^{2}\left(2 e^{\omega_{2}-\omega_{1}} \alpha_{o}\right)\left(\left(e^{4 \omega_{1}}+1\right) \cos ^{2}\left(2\left(\alpha_{o}-\Theta_{o}\right)\right)+e^{4 \omega_{1}}-1\right) \\
& \pm \sqrt{2}\left[-e^{8 \omega_{1}} \csc ^{4}\left(2\left(\alpha_{o}-\Theta_{o}\right)\right) \sin ^{2}\left(2\left(1-e^{\omega_{2}-\omega_{1}}\right) \alpha_{o}-2 \Theta_{o}\right)\left\{\left(1-e^{4 \omega_{1}}\right) \cos \left(4\left(\alpha_{o}-\Theta_{o}\right)\right)\right.\right. \\
& \left.\left.+e^{4 \omega_{1}} \cos \left(4\left(1-e^{\omega_{2}-\omega_{1}}\right) \alpha_{o}-4 \Theta_{o}\right)+\left(e^{4 \omega_{1}}-1\right)\left(e^{4 \omega_{1}} \cos \left(4 e^{\omega_{2}-\omega_{1}} \alpha_{o}\right)+1\right)-e^{8 \omega_{1}}\right\}\right]^{\frac{1}{2}} \\
& \left.\left.-e^{8 \omega_{1}} \sin ^{2}\left(2 e^{\omega_{2}-\omega_{1}} \alpha_{o}\right)+e^{4 \omega_{1}}\left(\cos ^{2}\left(2 e^{\omega_{2}-\omega_{1}} \alpha_{o}\right)+1\right)\right\}^{-1}\right]^{\frac{1}{2}}, \\
c_{o_{1}}= & 2\left[1+\left(\frac{e^{2 \omega_{1}} \sin \left(2 e^{\omega_{2}-\omega_{1}} \alpha_{o}\right)}{\sin \left(2\left(\Theta_{o}-\alpha_{o}\right)\right)}\right)^{2}\left(\left(\frac{c_{i_{1}}}{2 e^{2 \omega_{2}}}\right)^{2}-1\right)\right]^{\frac{1}{2}} \cdot
\end{aligned}
$$

Using (3.8), one finds

$$
h(\Theta)=\left\{\begin{array}{rlrl}
\tan ^{-1}\left[e ^ { - ( \omega _ { 1 } + \omega _ { 2 } ) } \left(-\sqrt{\left(\frac{1}{2} c_{i_{1}} e^{\omega_{1}-\omega_{2}}\right)^{2}-e^{2\left(\omega_{1}+\omega_{2}\right)}}\right.\right. & \\
\left.\left.+\frac{1}{2} c_{i_{1}} e^{\omega_{1}-\omega_{2}}\right) \tan \left(e^{\omega_{2}-\omega_{1}} \Theta+\frac{1}{2} c_{i_{2}}\right)\right]+c_{i_{3}}, & & \\
\tan ^{-1}\left[\left(\frac{1}{2} c_{o_{1}}-\sqrt{\frac{1}{4} c_{o_{1}}^{2}-1}\right) \tan \left(\Theta+\frac{1}{2} c_{o_{2}}\right)\right]+c_{o_{3}}, & \alpha_{o} \leq \Theta \leq \alpha_{o},
\end{array}\right.
$$

\footnotetext{
${ }^{3}$ The resultant force acting in the $\theta$-direction on the circular boundary is trivially zero as $\zeta^{\prime}(\Theta)$ is an odd function. In addition, $t^{z}=0$ as $P^{2 R}=0$.

${ }^{4}$ The volume form of a Riemannian manifold is defined as $\Omega=\sqrt{\operatorname{det}\left(g_{i j}\right)} d x^{1} \wedge d x^{2} \wedge \ldots \wedge d x^{n}$.

${ }^{5} \mathrm{Here}$, it suffices to specify $\zeta(\Theta)$ and $h(\Theta)$ only in the upper half region of the wedge as these functions are even and odd, respectively.
} 
where $c_{i_{3}}$ and $c_{o_{3}}$ are constants of integration. Imposing the condition $h(0)=0$, implies that $c_{i_{3}}=$ $-k_{2} \frac{\pi}{2}-k_{3} \pi$, where $k_{3} \in \mathbb{Z}$. Using the continuity of $h(\Theta)$ at $\Theta=\alpha_{o}$, we have

$$
\begin{array}{r}
c_{o_{3}}=\tan ^{-1}\left[e ^ { - ( \omega _ { 1 } + \omega _ { 2 } ) } \left(-\sqrt{\left(\frac{1}{2} c_{i_{1}} e^{\omega_{1}-\omega_{2}}\right)^{2}-e^{2\left(\omega_{1}+\omega_{2}\right)}}\right.\right. \\
\left.\left.+\frac{1}{2} c_{i_{1}} e^{\omega_{1}-\omega_{2}}\right) \tan \left(e^{\omega_{2}-\omega_{1}} \alpha_{o}+\frac{k_{2} \pi}{2}\right)\right] \\
-\tan ^{-1}\left[\left(\frac{1}{2} c_{o_{1}}-\sqrt{\frac{1}{4} c_{o_{1}}^{2}-1}\right) \tan \left(\alpha_{o}-\Theta_{o}+\frac{k_{1} \pi}{2}\right)\right] \\
-k_{2} \frac{\pi}{2}-k_{3} \pi .
\end{array}
$$

Remark 3.4. From (3.49) and (3.50), it can be seen that $\omega_{1}=0$ implies that $c_{i_{1}}=2 e^{2 \omega_{2}}$ and $c_{o_{1}}=2$. In this case, the radius of the wedge does not change, and the inclusion deforms independently of the matrix in the circumferential direction, such that $h(\Theta)=e^{\omega_{2}} \Theta$ in the inclusion, and $h(\Theta)=\left(e^{\omega_{2}}-1\right) \alpha_{o}+\Theta$ outside the inclusion. Furthermore, all the components of the stress tensor are zero point-wise.

Using (3.18), (3.37), (3.41), and (3.43), one finds the physical components of the Cauchy stress, along with the pressure field as follows

$$
\hat{\sigma}^{r r}=\left\{\begin{array}{cc}
\mu\left(e^{-2 \omega_{2}} \zeta^{\prime}(\Theta)^{2}+e^{-2 \omega_{1}} \zeta(\Theta)^{2}\right) & \\
-p_{i}, & |\Theta| \leq \alpha_{o}, \\
-p_{o}+\mu\left(\zeta^{\prime}(\Theta)^{2}+\zeta(\Theta)^{2}\right), & |\Theta|>\alpha_{o},
\end{array}\right.
$$

$$
\begin{aligned}
& \hat{\sigma}^{r \theta}= \begin{cases}\frac{\mu \zeta^{\prime}(\Theta)}{\zeta(\Theta)} e^{\omega_{1}-\omega_{2}}, & |\Theta| \leq \alpha_{o}, \\
\frac{\mu \zeta^{\prime}(\Theta)}{\zeta(\Theta)}, & |\Theta|>\alpha_{o},\end{cases} \\
& \hat{\sigma}^{\theta \theta}= \begin{cases}\frac{1}{\zeta(\Theta)^{2}}\left(\mu e^{2 \omega_{1}}-p_{i} \zeta(\Theta)^{2}\right), & |\Theta| \leq \alpha_{o}, \\
\frac{1}{\zeta(\Theta)^{2}}\left(\mu-p_{o} \zeta(\Theta)^{2}\right), & |\Theta|>\alpha_{o},\end{cases} \\
& \hat{\sigma}^{z z}= \begin{cases}-p_{i}+\mu, & |\Theta| \leq \alpha_{o}, \\
-p_{o}+\mu, & |\Theta|>\alpha_{o},\end{cases}
\end{aligned}
$$

where

$$
p(\Theta)= \begin{cases}p_{i}=\frac{\mu}{\zeta\left(\Theta_{o}\right)^{2}}+\frac{\mu\left(e^{2 \omega_{1}}-1\right)}{\zeta\left(\alpha_{o}\right)^{2}}, & |\Theta| \leq \alpha_{o}, \\ p_{o}=\frac{\mu}{\zeta\left(\Theta_{o}\right)^{2}}, & |\Theta|>\alpha_{o} .\end{cases}
$$

Remark 3.5. Note that the physical components of the Cauchy stress are independent of the radial coordinate $R$. Therefore, the stress components at the apex of the wedge do not have a unique value. In fact, this should not be surprising given that the eigenstrain distribution (3.35) is multi-valued at the apex.

Numerical results.. We now consider some specific examples and find the deformed shape of the wedge and the corresponding residual stress field. A comparison of the deformations and the distribution of the stress components for different values of eigenstrains $\omega_{1}$ and $\omega_{2}$, and various wedge geometries are presented in Figures 3 to 7. A wedge having an inclusion with positive pure dilatational eigenstrains is depicted in Figure 3. As expected both the inclusion and the matrix regions are pushed outward in the radial direction, with the matrix filaments stretched more than those of the inclusion. Although the circumferential eigenstrain is positive in this case, the total wedge angle is decreased. As a matter of fact, for any positive value of pure dilatational eigenstrains the angle of the wedge is reduced after deformation. Moreover, $\hat{\sigma}^{r r}$ is compressive in the inclusion and tensile in the matrix, and undergoes a jump at the inclusion-matrix interface, which is also the case as illustrated in other figures. ${ }^{6}$

For an inclusion with negative purely dilatational eigenstrains, all the radial planes of the wedge displace inward, with the matrix region being shortened more than the inclusion (Figure 4). Undeformed and deformed configurations of a wedge with positive radial and negative circumferential eigenstrains is shown in Figure 5. Note that $\hat{\sigma}^{\theta \theta}$ is tensile throughout the wedge, and $\hat{\sigma}^{r r}$ is compressive and tensile in the inclusion and the matrix, respectively. A wedge containing an inclusion with a negative radial and positive circumferential eigenstrains is shown in Figure 6. Notice that unlike other cases for which the deformation was purely inward or purely outward, in this example, the deformation is no longer unidirectional. In this example, the central region of the inclusion moves outward, while the region close to the inclusion-matrix interface moves inward. Moreover, this trend continues even for the large negative values of the radial eigenstrain. Although the circumferential eigenstrain is positive, the inclusion shrinks in the circumferential direction, while the matrix expands in this direction such that the total angle of the wedge is increased. Figure 7 shows an inclusion with a purely radial eigenstrain. Note that although the eigenstrian is purely radial, the wedge is deformed considerably in the circumferential direction, with the inclusion expanding and the matrix shrinking circumferentially such that the total angle of the wedge is reduced.

\footnotetext{
${ }^{6}$ Note that the undeformed (reference) configuration shown in the following figures has a metric different from that of the deformed configuration, and hence, the area of the body seen in the figures is not representative of the actual volume of the body in the (non-flat) reference configuration. In particular, the material manifold is equipped with the non-trivial Riemannian metric (3.3), giving a volume for the body in the reference configuration different from that given by the flat Euclidean metric.
} 

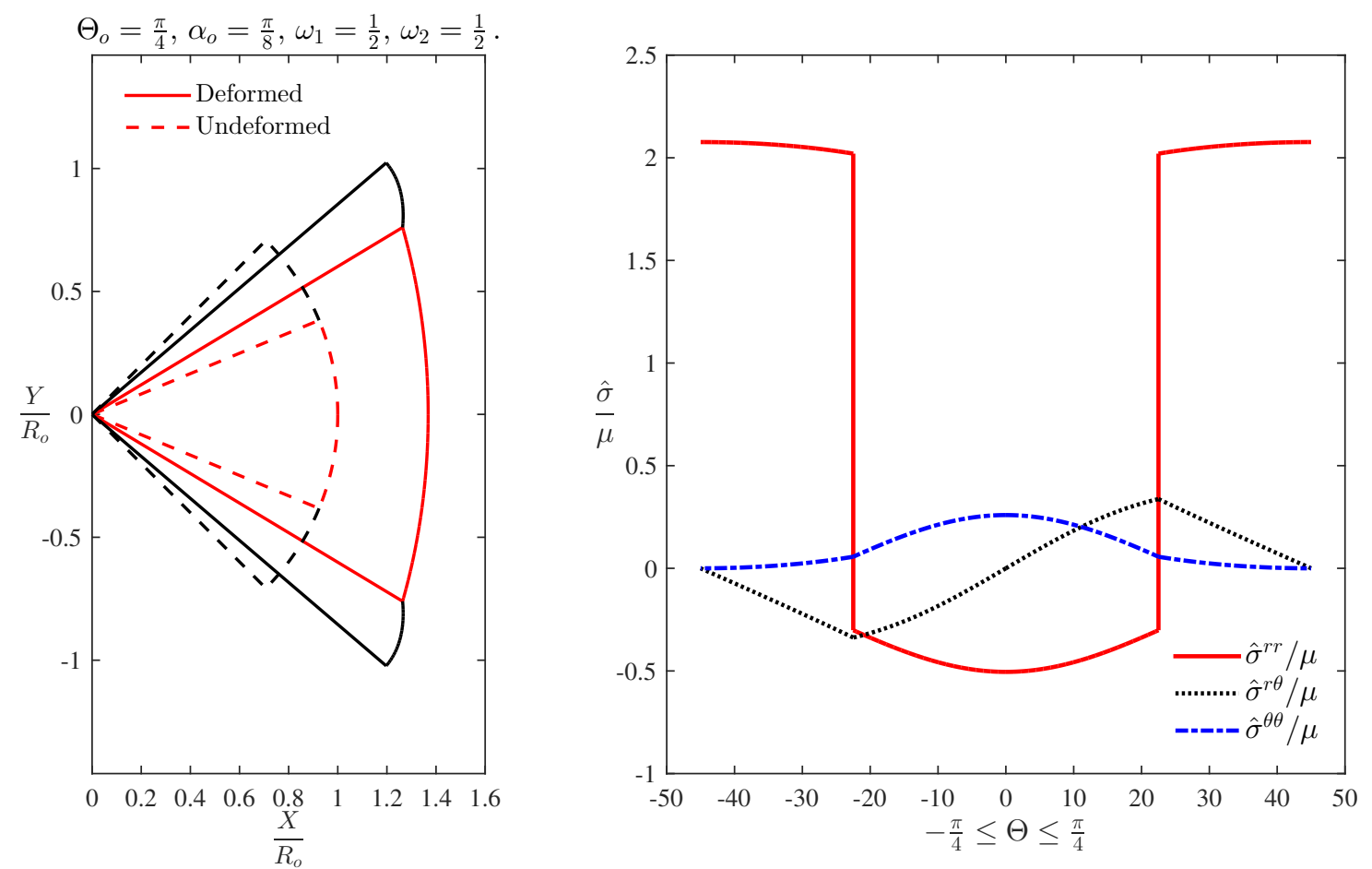

Figure 3: Left: The initial and deformed configurations of a wedge with the initial half angle $\Theta_{o}=\frac{\pi}{4}$ having an inclusion with $\alpha_{o}=\frac{\pi}{8}$ and the pure dilatational eigenstrain distribution $\omega_{1}=\omega_{2}=\frac{1}{2}$. Right: Variation of the physical components of the Cauchy stress tensor versus $\Theta$.
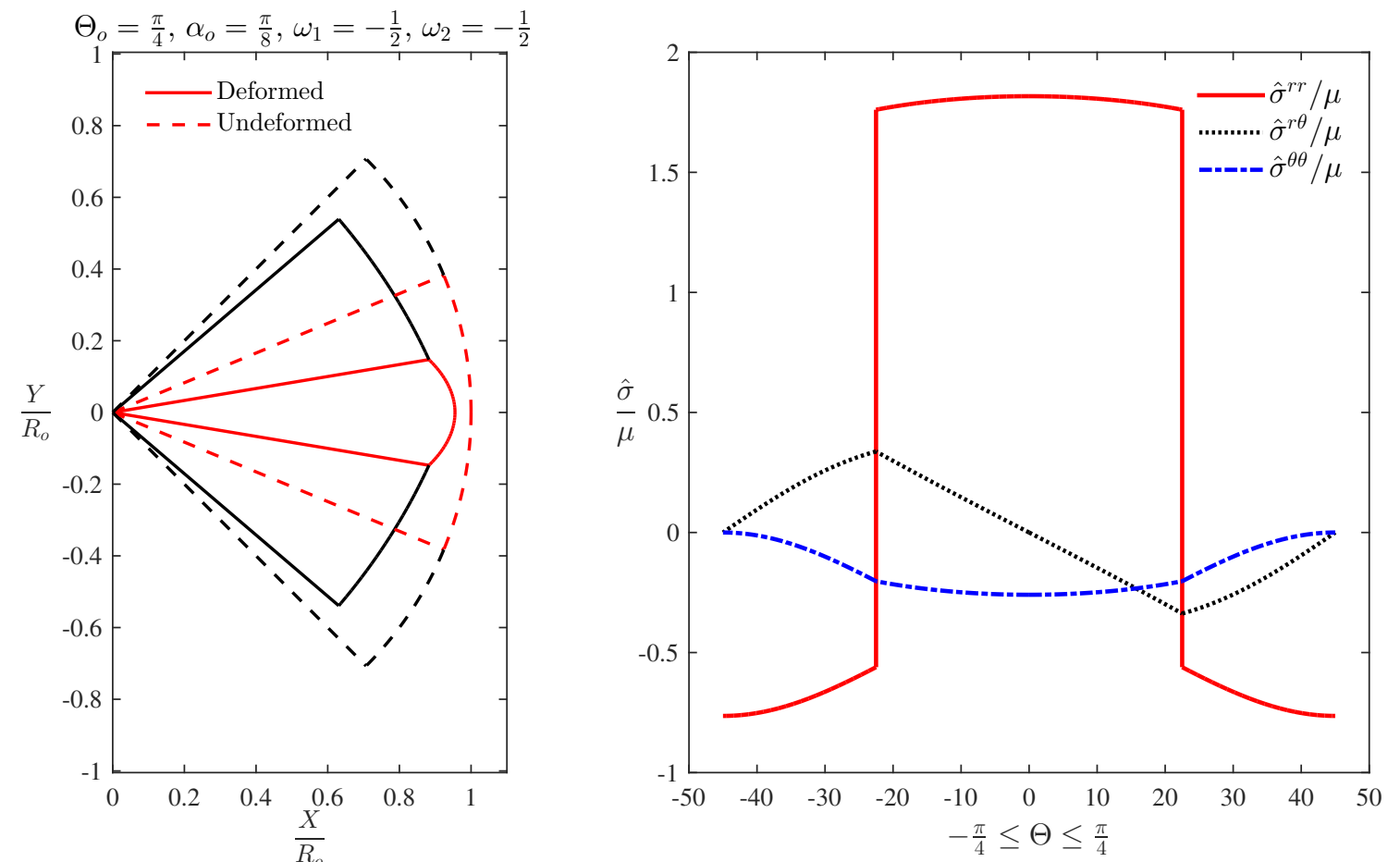

Figure 4: Left: The initial and deformed configurations of a wedge with the initial half angle $\Theta_{o}=\frac{\pi}{4}$ having an inclusion with $\alpha_{o}=\frac{\pi}{8}$ and the pure dilatational eigenstrain distribution $\omega_{1}=\omega_{2}=-\frac{1}{2}$. Right: Variation of the physical components of the Cauchy stress tensor versus $\Theta$. 

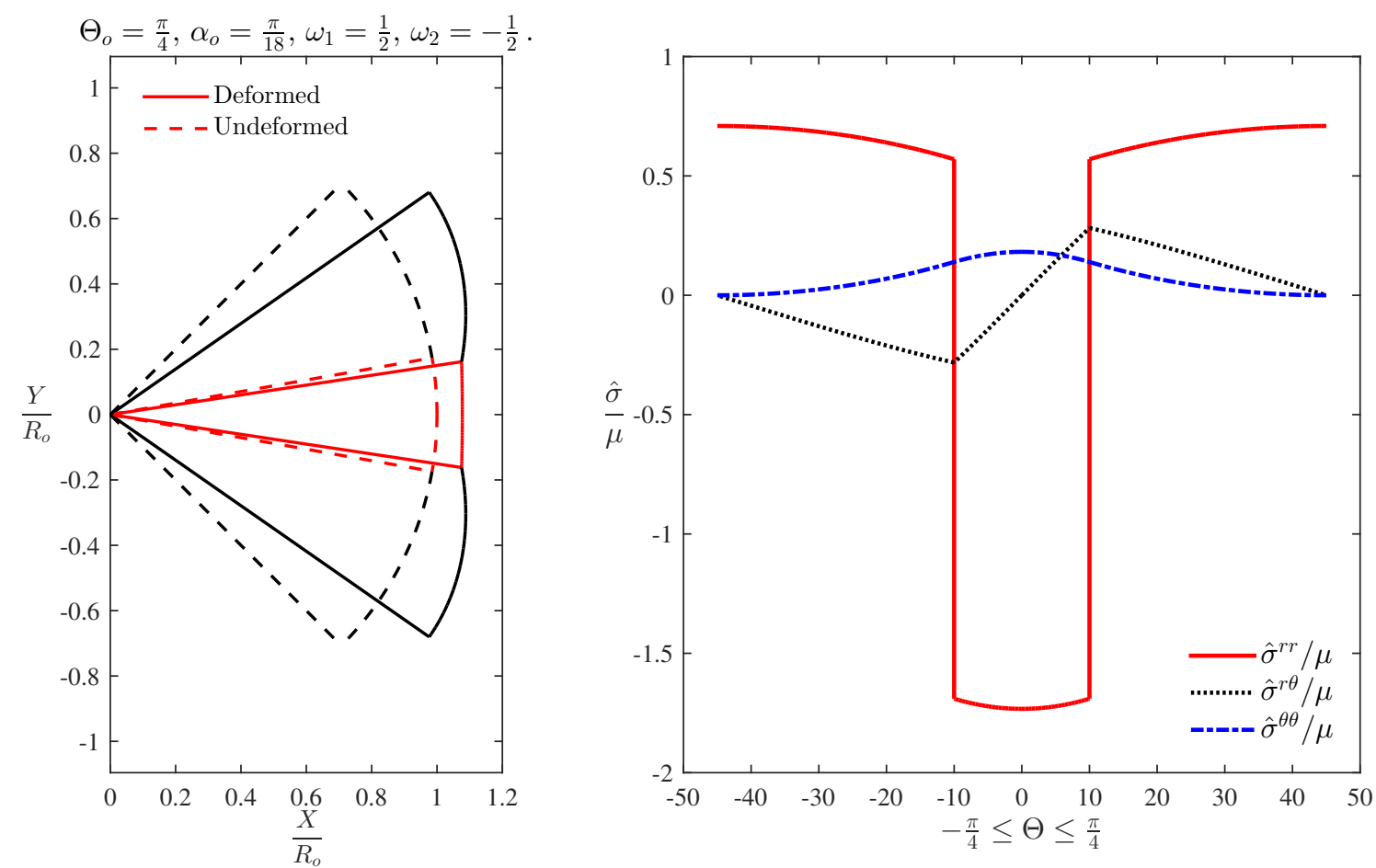

Figure 5: Left: The initial and deformed configurations of a wedge with the initial half angle $\Theta_{o}=\frac{\pi}{4}$ having an inclusion with $\alpha_{o}=\frac{\pi}{18}$ and the constant eigenstrain distribution $\omega_{1}=\frac{1}{2}$ and $\omega_{2}=-\frac{1}{2}$. Right: Variation of the physical components of the Cauchy stress tensor versus $\Theta$.
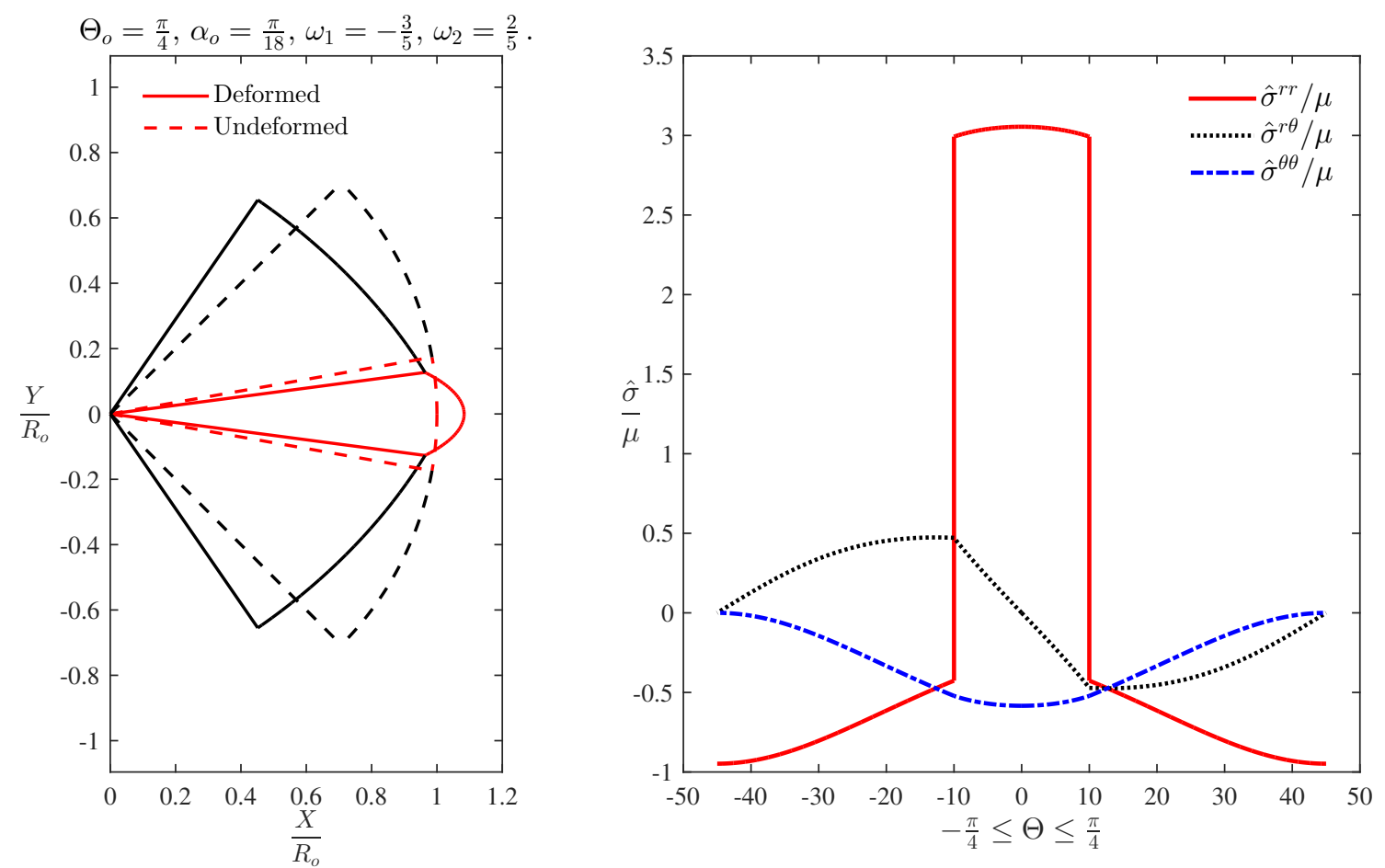

Figure 6: Left: The initial and deformed configurations of a wedge with the initial half angle $\Theta_{o}=\frac{\pi}{4}$ having an inclusion with $\alpha_{o}=\frac{\pi}{18}$ and the constant eigenstrain distribution $\omega_{1}=-\frac{3}{5}$ and $\omega_{2}=\frac{2}{5}$. Right: Variation of the physical components of the Cauchy stress tensor versus $\Theta$. In this example, the deformation is non-unidirectional. 

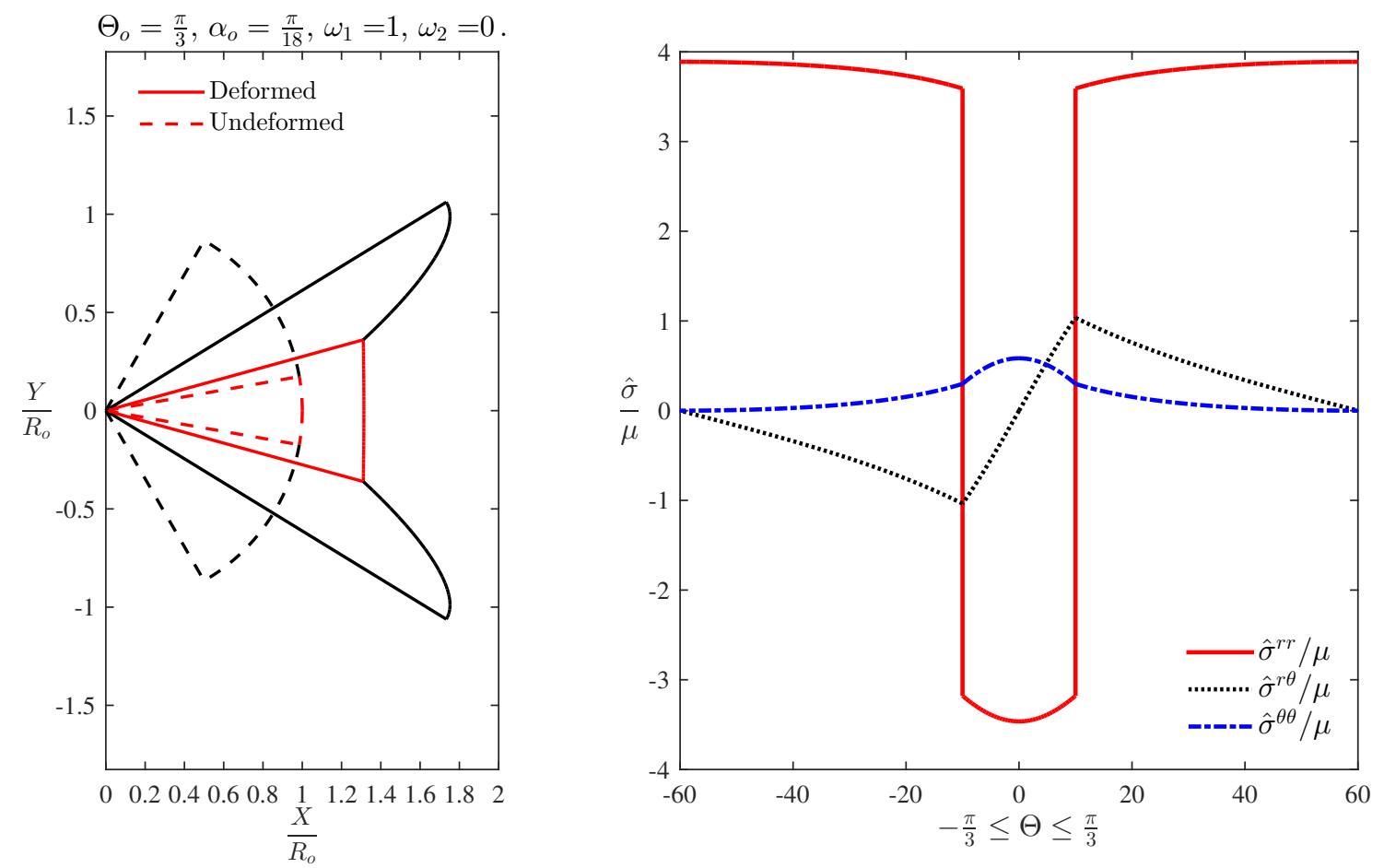

Figure 7: Left: The initial and deformed configurations of a wedge with the initial half angle $\Theta_{o}=\frac{\pi}{3}$ having an inclusion with $\alpha_{o}=\frac{\pi}{18}$ and the constant eigenstrain distribution $\omega_{1}=1$ and $\omega_{2}=0$. Right: Variation of the physical components of the Cauchy stress tensor versus $\Theta$.

\subsection{A Mooney-Rivlin inhomogeneity with uniform} eigenstrains in a neo-Hookean wedge with clamped lateral boundaries

In this example, we consider an inhomogeneity made of a Mooney-Rivlin material in a neo-Hookean wedge with fixed (clamped) lateral boundaries such that they cannot move in the radial and circumferential directions. The energy function has the following $\Theta$-dependence in the wedge

$W\left(I_{1}, I_{2}, \Theta\right)= \begin{cases}\frac{\mu_{1}}{2}\left(I_{1}-3\right)+\frac{\mu_{2}}{2}\left(I_{2}-3\right), & |\Theta| \leq \alpha_{o}, \\ \frac{\mu_{o}}{2}\left(I_{1}-3\right), & |\Theta|>\alpha_{o} .\end{cases}$

Moreover, we consider the eigenstrain distribution in the wedge given by (3.35). Looking at (3.33) and (3.34) one observes that equations (3.37) and (3.38) of Section 3.1 hold for this example as well if $\mu$ in (3.38) is replaced by $\mu_{1}+\mu_{2}$ and $\mu_{o}$ in the inhomogeneity and the matrix, respectively. Therefore

$$
p(R, \Theta)= \begin{cases}f_{o} \ln (R \zeta(\Theta))+p_{i}, & |\Theta| \leq \alpha_{o}, \\ f_{o} \ln (R \zeta(\Theta))+p_{o}, & |\Theta|>\alpha_{o}\end{cases}
$$

and

$$
\zeta^{\prime}(\Theta)^{2}=\left\{\begin{array}{c}
c_{i_{1}}+\frac{2 f_{o} e^{2 \omega_{2}}}{\mu_{1}+\mu_{2}} \ln \zeta(\Theta)-\zeta^{2}(\Theta) e^{2\left(\omega_{2}-\omega_{1}\right)} \\
-\frac{e^{2\left(\omega_{1}+\omega_{2}\right)}}{\zeta^{2}(\Theta)}, \quad|\Theta| \leq \alpha_{o} \\
c_{o_{1}}+\frac{2 f_{o}}{\mu_{o}} \ln \zeta(\Theta)-\zeta^{2}(\Theta) \\
-\frac{1}{\zeta^{2}(\Theta)}, \quad|\Theta|>\alpha_{o}
\end{array}\right.
$$

Boundary conditions.. The continuity of the traction vector at the inhomogeneity-matrix interface implies that

$$
\left.\frac{\mu_{1}+\mu_{2}}{\mu_{o}} e^{\omega_{1}-\omega_{2}} \zeta^{\prime}(\Theta)\right|_{\Theta=\alpha_{o}^{-}}=\left.\zeta^{\prime}(\Theta)\right|_{\Theta=\alpha_{o}^{+}},
$$

and

$$
\frac{\mu_{1}+\mu_{2}}{\mu_{o}} e^{2 \omega_{1}}-1=\frac{p_{i}-p_{o}-\mu_{2}}{\mu_{o}} \zeta\left( \pm \alpha_{o}\right)^{2} .
$$

We assume that the lateral boundaries of the wedge are clamped, i.e. ${ }^{7}$

$$
\zeta\left(\Theta_{o}\right)=1, \quad h\left(\Theta_{o}\right)=\Theta_{o} .
$$

In order to determine the pressure constants $p_{i}$ and $p_{o}$, we assume that the resultant force acting on the circular boundary of the wedge vanishes. Using (3.46), the radial force per unit length of the cylinder in the $Z$ direction is simplified to read

$$
\begin{gathered}
F_{r}=2 \mu_{o} R_{o}\left[e ^ { \omega _ { 1 } + \omega _ { 2 } } \int _ { 0 } ^ { \alpha _ { o } } \left\{e^{-2 \omega_{1}}\left(\frac{\mu_{1}}{\mu_{o}}+\frac{\mu_{2}}{\mu_{o}}\right) \zeta(\Theta)\right.\right. \\
\left.-\frac{f_{o}}{\mu_{o}} \frac{\ln \left(R_{o} \zeta(\Theta)\right)}{\zeta(\Theta)}-\left(\frac{p_{i}}{\mu_{o}}-\frac{\mu_{2}}{\mu_{o}}\right) \frac{1}{\zeta(\Theta)}\right\} d \Theta \\
\left.+\int_{\alpha_{o}}^{\Theta_{o}}\left\{\zeta(\Theta)-\frac{f_{o}}{\mu_{o}} \frac{\ln \left(R_{o} \zeta(\Theta)\right)}{\zeta(\Theta)}-\frac{p_{o}}{\mu_{o} \zeta(\Theta)}\right\} d \Theta\right] .
\end{gathered}
$$

We proceed to numerically solve the boundary-value problem (3.60) along with the above boundary conditions and the constraint of a zero-boundary resultant. The physical components of the Cauchy stress read

\footnotetext{
${ }^{7}$ The functions $\zeta(\Theta)$ and $h(\Theta)$ are even and odd, respectively, and hence, $\zeta^{\prime}(0)=0$ and $h(0)=0$.
} 


$$
\begin{aligned}
& \hat{\sigma}^{r r}= \begin{cases}-f_{o} \ln (R \zeta(\Theta))-p_{i}+\left(\mu_{1}+\mu_{2}\right)\left(e^{-2 \omega_{2}} \zeta^{\prime}(\Theta)^{2}+e^{-2 \omega_{1}} \zeta(\Theta)^{2}\right)+\mu_{2}, & |\Theta| \leq \alpha_{o}, \\
-f_{o} \ln (R \zeta(\Theta))-p_{o}+\mu_{o}\left(\zeta^{\prime}(\Theta)^{2}+\zeta(\Theta)^{2}\right), & |\Theta|>\alpha_{o},\end{cases} \\
& \hat{\sigma}^{r \theta}= \begin{cases}\frac{\left(\mu_{1}+\mu_{2}\right) \zeta^{\prime}(\Theta)}{\zeta^{\prime(\Theta)}} e^{\omega_{1}-\omega_{2}}, & |\Theta| \leq \alpha_{o}, \\
\frac{\mu_{o} \zeta^{\prime}(\Theta)}{\zeta(\Theta)}, & |\Theta|>\alpha_{o},\end{cases} \\
& \hat{\sigma}^{\theta \theta}= \begin{cases}\frac{1}{\zeta(\Theta)^{2}}\left(\left(\mu_{1}+\mu_{2}\right) e^{2 \omega_{1}}-\zeta(\Theta)^{2}\left(f_{o} \ln (R \zeta(\Theta))+p_{i}-\mu_{2}\right)\right), & |\Theta| \leq \alpha_{o}, \\
\frac{1}{\zeta(\Theta)^{2}}\left(\mu_{o}-\zeta(\Theta)^{2}\left(f_{o} \ln (R \zeta(\Theta))+p_{o}\right)\right), & |\Theta|>\alpha_{o},\end{cases} \\
& \hat{\sigma}^{z z}= \begin{cases}-f_{o} \ln (R \zeta(\Theta))-p_{i}+\mu_{2}\left(e^{-2 \omega_{2}} \zeta^{\prime}(\Theta)^{2}+e^{-2 \omega_{1}} \zeta(\Theta)^{2}+\frac{e^{2 \omega_{1}}}{\zeta(\Theta)^{2}}\right)+\mu_{1}, & |\Theta| \leq \alpha_{o}, \\
-f_{o} \ln (R \zeta(\Theta))-p_{o}+\mu_{o}, & |\Theta|>\alpha_{o},\end{cases}
\end{aligned}
$$

Remark 3.6. Note that $\hat{\sigma}^{r \theta}$ depends only on $\Theta$. Moreover, the radial dependence of $\hat{\sigma}^{r r}, \hat{\sigma}^{\theta \theta}$, and $\hat{\sigma}^{z z}$ is linear with respect to $\ln R$. We use this property and plot the stress components at $R=R_{o}$.

Numerical results.. The deformation of the wedge and the variation of the stress components for various eigenstrain distributions in the inhomogeneity with different elastic constants are examined and are presented in Figures 8 to 12. A wedge containing an inhomogeneity stiffer than the matrix with positive eigenstrains such that the circumferential eigenstrain is twice the radial one is shown in Figure 8. As expected all the radial planes of the wedge displace outward, with the inhomogeneity expanding more than the matrix. Furthermore, on the circular boundary $\hat{\sigma}^{r r}$ is negative in the inhomogeneity, positive in the matrix, and discontinuous at the inhomogeneity-matrix interface. Note that $\hat{\sigma}^{\theta \theta}$ is compressive almost everywhere on the circular boundary except for some small regions close to the lateral boundaries.

Figure 9 depicts an inhomogeneity placed in a stiffer matrix with anisotropic eigenstrains such that the radial eigenstrain is twice the circumferential one. It is observed that $\hat{\sigma}^{\theta \theta}$ is tensile on the circular boundary. Moreover, $\hat{\sigma}^{\theta \theta}$ and $\hat{\sigma}^{r r}$ are almost uniform in the inhomogeneity. For a wedge having an inhomogeneity stiffer than the matrix with negative circumferentially dominated eigenstrains, all the radial planes are contracted. In addition, $\hat{\sigma}^{r r}$ and $\hat{\sigma}^{\theta \theta}$ are almost uniform, and $\hat{\sigma}^{r \theta}$ is almost zero in the inhomogeneity (Figure 10).

Inhomogeneities with purely radial and purely circumferential eigenstrains are shown in Figures 11 and 12, respectively. For both cases, all the radial planes are elongated, with the inhomogeneity expanded and the matrix shrunk in the circumferential direction. Interestingly, the circumferential deformation is more pronounced in the purely radial eigenstrain case while radial deformation is more pronounced in the purely circumferential eigenstrain case. ${ }^{8}$ Unlike wedges with traction-free lateral boundaries for which a purely circumferential eigenstrain does not induce any residual stresses in the wedge, here residual stress is developed due to a purely circumferential eigenstrain because the wedge can no longer move freely in the circumferential direction. Note that $\hat{\sigma}^{r r}$ and $\hat{\sigma}^{\theta \theta}$ are almost uniform in the inhomogeneity for the purely radial eigenstrain case, with $\hat{\sigma}^{r r}$ undergoing a jump at the inhomogeneity-matrix interface. For the purely circumferential eigenstrain case, however, the stress components exhibit a different behavior in the inhomogeneity. For instance, $\hat{\sigma}^{r r}$ remains continuous at the inhomogeneity-matrix interface and does not tend to be uniform in the inhomogeneity.

\subsection{Stress-free eigenstrain distributions in a wedge}

In this section, we find those eigenstrain distributions that induce no residual stresses. For such eigenstrain distributions, the material manifold can be isometrically embedded into the ambient space, i.e, $\boldsymbol{G}=\varphi^{*} \boldsymbol{g} .{ }^{9} \quad$ Hence, for a simply-connected body, a stress-free eigenstrain distribution corresponds to a material metric with vanishing Riemannian curvature. Note that the wedge is a simply-connected body. Curvature tensor has the following components

$$
\begin{array}{r}
R_{B C D}^{A}=\frac{\partial \Gamma_{D B}^{A}}{\partial X^{C}}-\frac{\partial \Gamma_{C B}^{A}}{\partial X^{D}}+\Gamma_{C E}^{A} \Gamma_{D B}^{E} \\
-\Gamma_{D E}^{A} \Gamma_{C B}^{E},
\end{array}
$$

where the Christoffel symbols are defined as

$$
\Gamma_{B C}^{A}=\frac{1}{2} G^{A K}\left(\frac{\partial G_{K B}}{\partial X^{C}}+\frac{\partial G_{K C}}{\partial X^{B}}-\frac{\partial G_{B C}}{\partial X^{K}}\right) .
$$

For the material metric of the wedge, the Christoffel symbol matrices read

\footnotetext{
${ }^{8} \mathrm{~A}$ similar observation was made for the wedge with traction-free lateral boundaries having an inclusion with a purely radial eigenstrain (cf. Figure 7).

${ }^{9}$ Equivalently, the Lagrangian strain tensor must vanish.
} 
$\Theta_{o}=\frac{\pi}{4}, \alpha_{o}=\frac{\pi}{8}, \omega_{1}=\frac{1}{10}, \omega_{2}=\frac{2}{10}, \frac{\mu_{1}}{\mu_{o}}=1, \frac{\mu_{2}}{\mu_{o}}=1$.
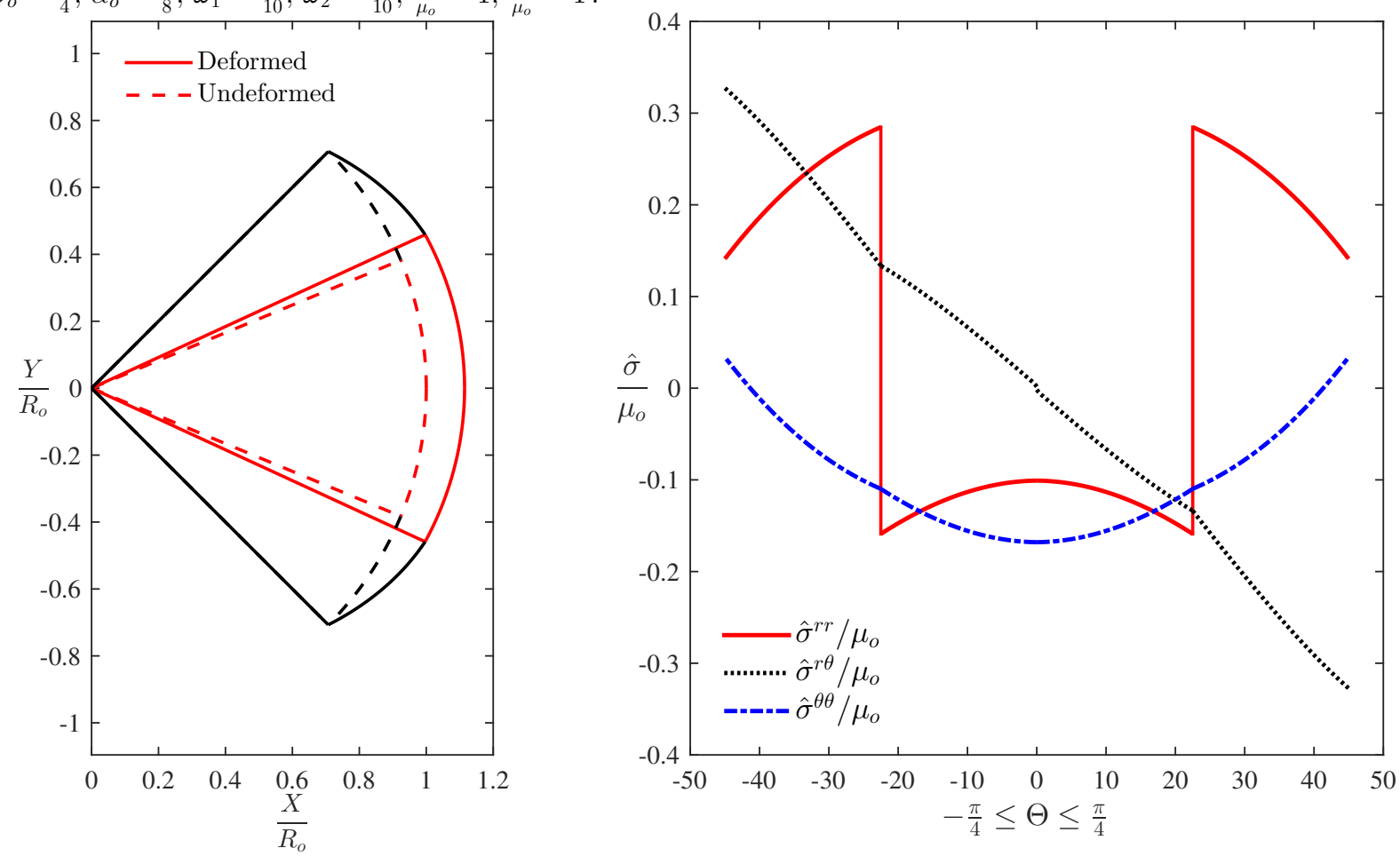

Figure 8: Left: The initial and deformed configurations of a wedge with fixed lateral boundaries and the initial half angle $\Theta_{o}=\frac{\pi}{4}$ having an inhomogenity with $\alpha_{o}=\frac{\pi}{8}, \frac{\mu_{1}}{\mu_{o}}=\frac{\mu_{2}}{\mu_{o}}=1$, and the constant eigenstrain distribution $\omega_{1}=\frac{1}{10}$ and $\omega_{2}=\frac{2}{10} .\left(\frac{f_{o}}{\mu_{o}}=-0.2761, \frac{p_{i}}{\mu_{o}}=3.1646\right)$. Right: Variation of the physical components of the Cauchy stress tensor versus $\Theta$ at $R=R_{o}$.

$\Theta_{o}=\frac{\pi}{4}, \alpha_{o}=\frac{\pi}{8}, \omega_{1}=1, \omega_{2}=\frac{1}{2}, \frac{\mu_{1}}{\mu_{o}}=\frac{1}{4}, \frac{\mu_{2}}{\mu_{o}}=\frac{1}{4}$.
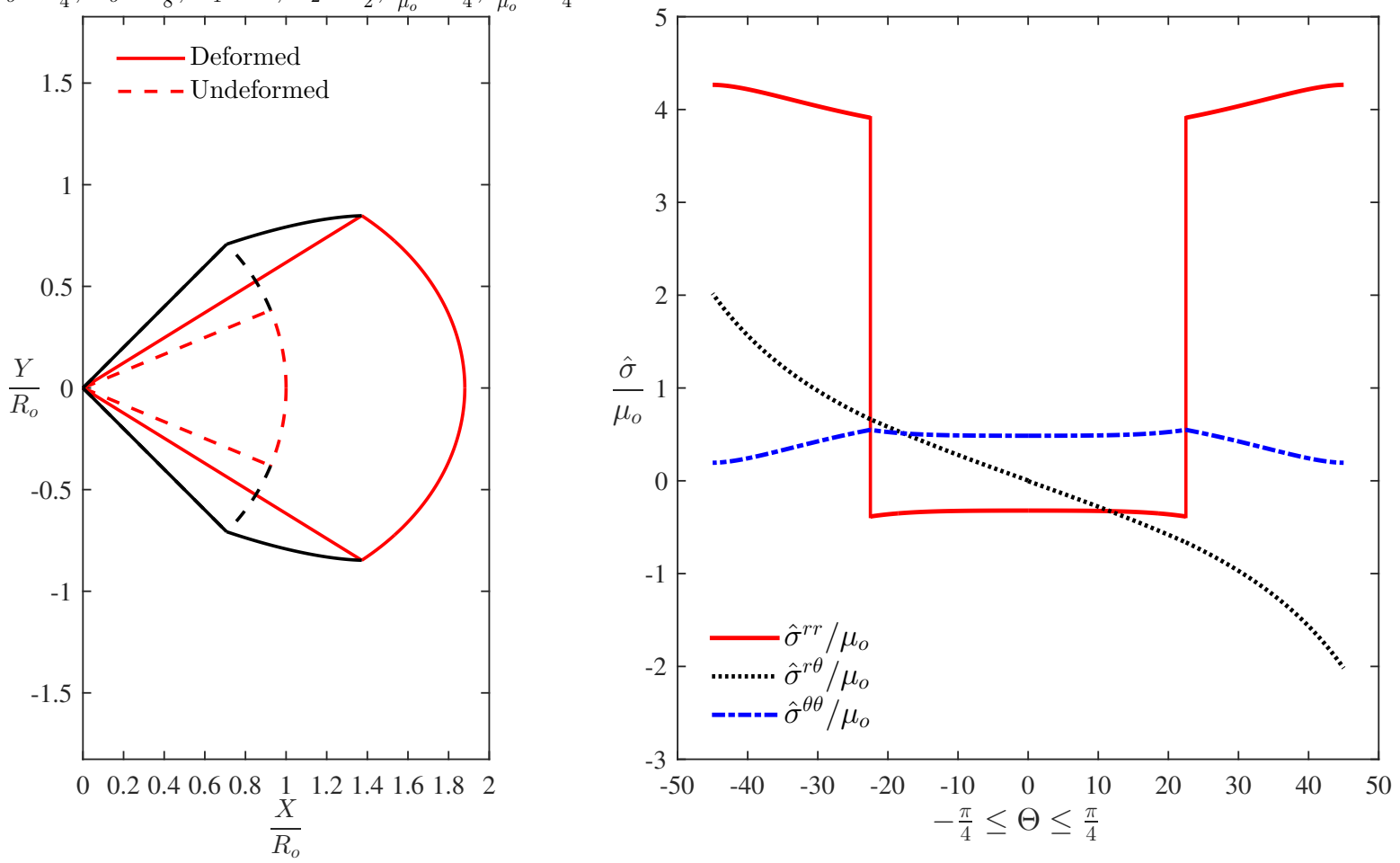

Figure 9: Left: The initial and deformed configurations of a wedge with fixed lateral boundaries and the initial half angle $\Theta_{o}=\frac{\pi}{4}$ having an inhomogenity with $\alpha_{o}=\frac{\pi}{8}, \frac{\mu_{1}}{\mu_{o}}=\frac{\mu_{2}}{\mu_{o}}=\frac{1}{4}$, and the constant eigenstrain distribution $\omega_{1}=1$ and $\omega_{2}=\frac{1}{2}$. $\left(\frac{f_{o}}{\mu_{o}}=-2.0293, \frac{p_{i}}{\mu_{o}}=2.0909\right)$. Right: Variation of the physical components of the Cauchy stress tensor versus $\Theta$ at $R=R_{o}$. 
$\Theta_{o}=\frac{\pi}{4}, \alpha_{o}=\frac{\pi}{8}, \omega_{1}=-\frac{1}{2}, \omega_{2}=-1, \frac{\mu_{1}}{\mu_{o}}=1, \frac{\mu_{2}}{\mu_{o}}=1$.
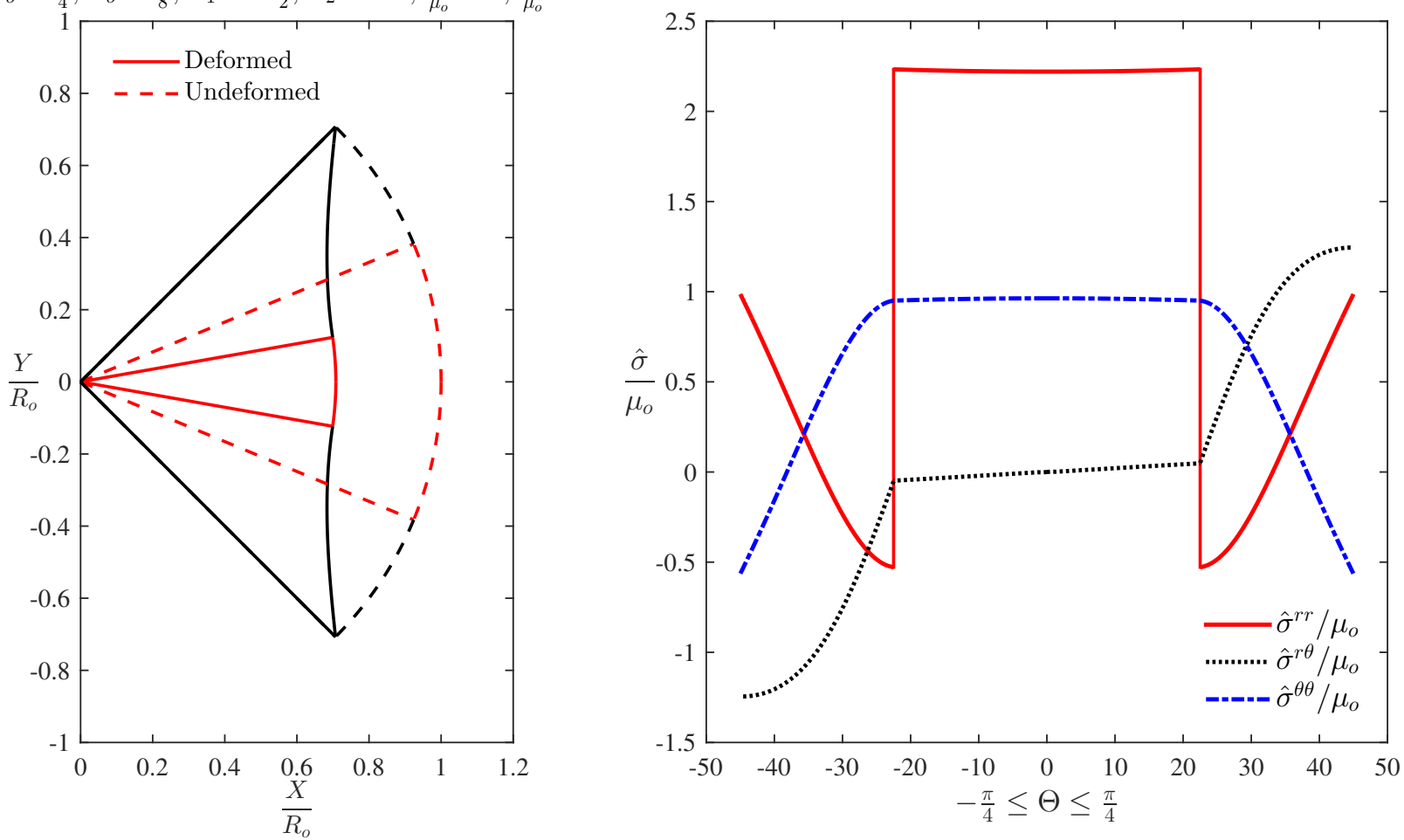

Figure 10: Left: The initial and deformed configurations of a wedge with fixed lateral boundaries and the initial half angle $\Theta_{o}=\frac{\pi}{4}$ having an inhomogenity with $\alpha_{o}=\frac{\pi}{8}, \frac{\mu_{1}}{\mu_{o}}=\frac{\mu_{2}}{\mu_{o}}=1$, and the constant eigenstrain distribution $\omega_{1}=-\frac{1}{2}$ and $\omega_{2}=-1 .\left(\frac{f_{o}}{\mu_{o}}=1.5500, \frac{p_{i}}{\mu_{o}}=2.0399\right)$. Right: Variation of the physical components of the Cauchy stress tensor versus $\Theta$ at $R=R_{o}$.

$\Theta_{o}=\frac{\pi}{4}, \alpha_{o}=\frac{\pi}{8}, \omega_{1}=1, \omega_{2}=0, \frac{\mu_{1}}{\mu_{o}}=\frac{1}{2}, \frac{\mu_{2}}{\mu_{o}}=\frac{1}{2}$.
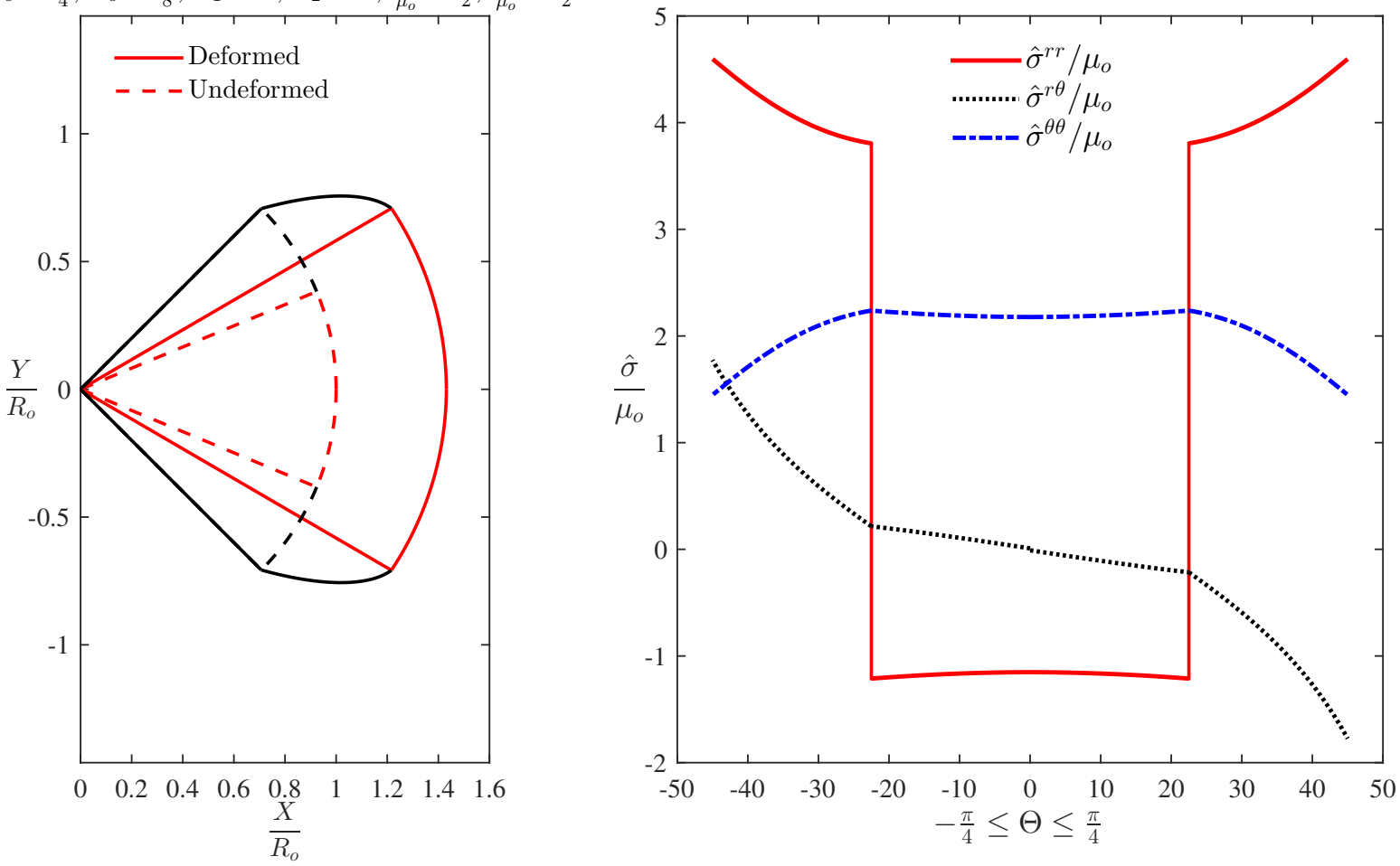

Figure 11: Left: The initial and deformed configurations of a wedge with fixed lateral boundaries and the initial half angle $\Theta_{o}=\frac{\pi}{4}$ having an inhomogenity with $\alpha_{o}=\frac{\pi}{8}, \frac{\mu_{1}}{\mu_{o}}=\frac{\mu_{2}}{\mu_{o}}=\frac{1}{2}$, and the constant eigenstrain distribution $\omega_{1}=1$ and $\omega_{2}=0 .\left(\frac{f_{o}}{\mu_{o}}=-3.7629, \frac{p_{i}}{\mu_{o}}=3.2785\right)$. Right: Variation of the physical components of the Cauchy stress tensor versus $\Theta$ at $R=R_{o}$. 

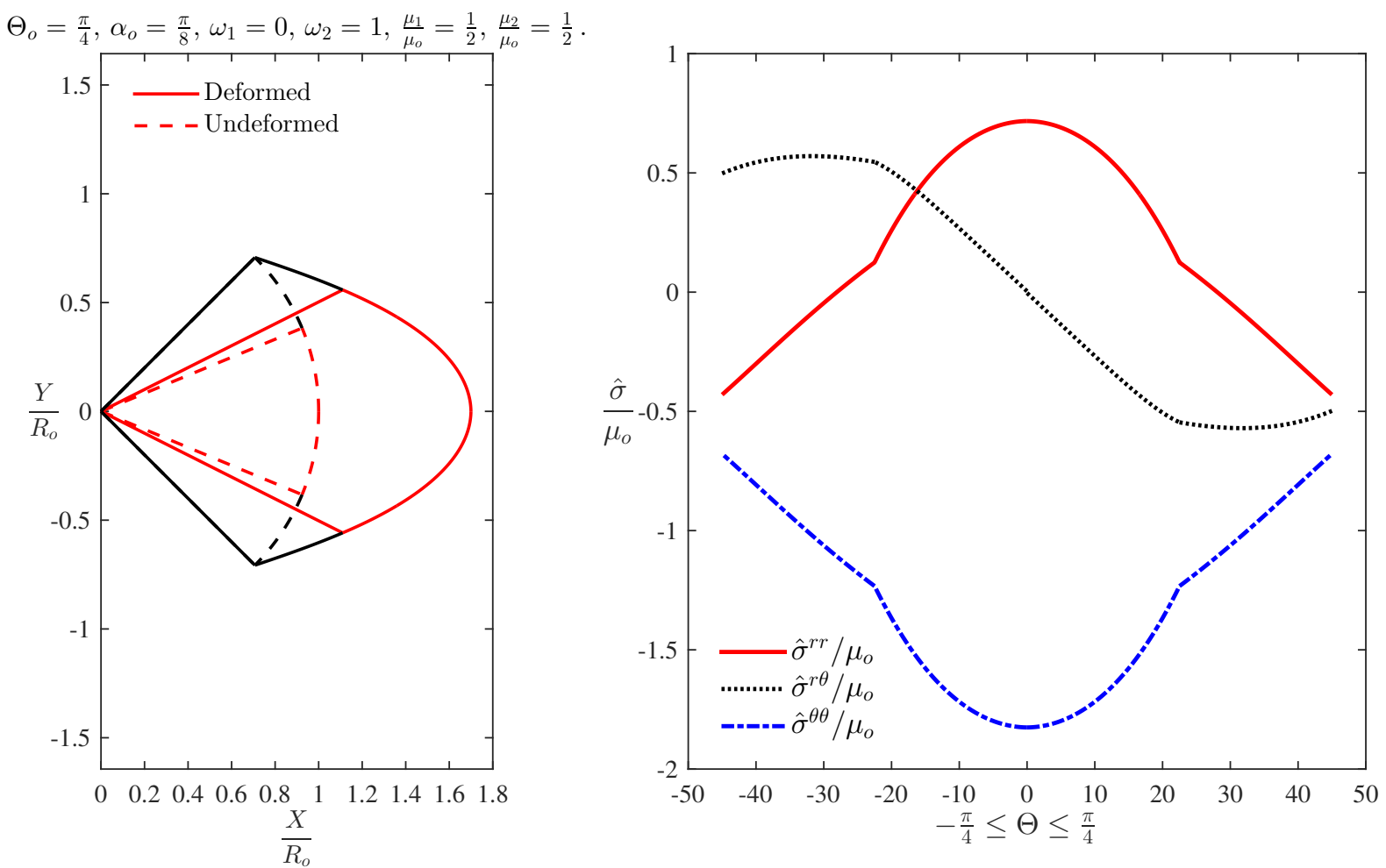

Figure 12: Left: The initial and deformed configurations of a wedge with fixed lateral boundaries and the initial half angle $\Theta_{o}=\frac{\pi}{4}$ having an inhomogenity with $\alpha_{o}=\frac{\pi}{8}, \frac{\mu_{1}}{\mu_{o}}=\frac{\mu_{2}}{\mu_{o}}=\frac{1}{2}$, and the constant eigenstrain distribution $\omega_{1}=0$ and $\omega_{2}=1 .\left(\frac{f_{o}}{\mu_{o}}=0.9305, \frac{p_{i}}{\mu_{o}}=2.1780\right)$. Right: Variation of the physical components of the Cauchy stress tensor versus $\Theta$ at $R=R_{o}$.

$$
\begin{aligned}
& \Gamma^{R}=\left[\Gamma_{A B}^{R}\right]=\left(\begin{array}{ccc}
0 & \omega_{R}^{\prime}(\Theta) & 0 \\
\omega_{R}^{\prime}(\Theta) & -\operatorname{Re}^{2\left(\omega_{\Theta}(\Theta)-\omega_{R}(\Theta)\right)} & 0 \\
0 & 0 & -\operatorname{Re}^{2\left(\omega_{\Theta}(\Theta)-\omega_{R}(\Theta)\right)} \sin ^{2} \Theta
\end{array}\right), \\
& \Gamma^{\Theta}=\left[\Gamma_{A B}^{\Theta}\right]=\left(\begin{array}{ccc}
-\frac{1}{R^{2}} e^{2\left(\omega_{R}(\Theta)-\omega_{\Theta}(\Theta)\right)} \omega_{R}^{\prime}(\Theta) & \frac{1}{R} & 0 \\
\frac{1}{R} & \omega_{\Theta}^{\prime}(\Theta) & 0 \\
0 & 0 & -\sin \Theta\left[\cos \Theta+\sin \Theta \omega_{\Theta}^{\prime}(\Theta)\right]
\end{array}\right), \\
& \Gamma^{\Phi}=\left[\Gamma_{A B}^{\Phi}\right]=\left(\begin{array}{ccc}
0 & 0 & \frac{1}{R} \\
0 & 0 & \cot \Theta+\omega_{\Theta}^{\prime}(\Theta) \\
\frac{1}{R} & \cot \Theta+\omega_{\Theta}^{\prime}(\Theta) & 0
\end{array}\right) .
\end{aligned}
$$

The non-trivially non-zero components of the curvature tensor for the cylinder with the metric $\boldsymbol{G}$ are

$$
\begin{aligned}
R_{\Theta R R}^{\Theta}=-R_{R \Theta R}^{\Theta}=\frac{e^{2\left(\omega_{R}(\Theta)-\omega_{\Theta}(\Theta)\right)}}{R^{2}}\left[-\omega_{\Theta}^{\prime}(\Theta) \omega_{R}^{\prime}(\Theta)\right. \\
\left.+\omega_{R}^{\prime}(\Theta)^{2}+\omega_{R}^{\prime \prime}(\Theta)\right], \\
R_{R \Theta \Theta}^{R}=-R_{\Theta R \Theta}^{R}=-\omega_{\Theta}^{\prime}(\Theta) \omega_{R}^{\prime}(\Theta)+\omega_{R}^{\prime}(\Theta)^{2} \\
+\omega_{R}^{\prime \prime}(\Theta) .
\end{aligned}
$$

Therefore, in order for an eigenstrain distribution to be stress-free in a wedge, it needs to satisfy the following non-linear ordinary differential equation:

$$
-\omega_{\Theta}^{\prime}(\Theta) \omega_{R}^{\prime}(\Theta)+\omega_{R}^{\prime}(\Theta)^{2}+\omega_{R}^{\prime \prime}(\Theta)=0
$$

Using this ODE, given $\omega_{\Theta}(\Theta), \omega_{R}(\Theta)$ is expressed as

$$
\omega_{R}(\Theta)=c_{2}+\ln \left(c_{1}+\int_{0}^{\Theta} e^{\omega_{\Theta}(\phi)} d \phi\right) .
$$

Remark 3.7. In the special case of $\omega=\omega_{R}(\Theta)=$ $\omega_{\Theta}(\Theta)$, we have a linear solution $\left(\omega(\Theta)=c_{1} \Theta+c_{2}\right)$ for the stress-free eigenstrain distribution, where $c_{1}$ and $c_{2}$ are constants.

\section{Conclusions}

In this paper we studied the residual stress field generated by a circumferentially-symmetric distribution of finite eigenstrains in an incompressible, isotropic elastic wedge. Using a semi-inverse method 
by assuming a specific class of deformations, we solved for the deformation and stress fields in the wedge for an arbitrary circumferentially-symmetric distribution of finite eigenstrains. We solved two examples. In the first one, we considered an inclusion with uniform eigenstrains in a neo-Hookean wedge with traction-free lateral boundaries and obtained exact solutions for the residual stress and deformation fields. We observed that if the eigenstrain distribution is purely circumferential, the pressure field remains continuous at the inclusion-matrix interface and the stress tensor is zero everywhere. Moreover, we observed that the deformation of the wedge fails to be unidirectional for an inclusion with a negative radial $\left(\omega_{1}<0\right)$ and positive circumferential $\left(\omega_{2}>0\right)$ eigenstrians even for large negative values of the radial eigenstrain. Furthermore, we found that the total wedge angle is reduced for any value of pure dilatational eigenstrains. In the second example, we considered a neo-Hookean wedge with clamped lateral boundaries having a symmetric Mooney-Rivlin inhomogeneity with uniform eigenstrains. We examined several cases of eigenstrain distributions for different relative stiffnesses of the inhomogeneity and the matrix. We observed that the circumferential and radial deformations are more pronounced in wedges containing inhomogeneities with only radial and only circumferential eigenstrains. In addition, we noticed that for a pure radial eigenstrain distribution, $\hat{\sigma}^{r r}$ and $\hat{\sigma}^{\theta \theta}$ are almost uniform in the inhomogeneity, and $\hat{\sigma}^{r r}$ has a jump at the inhomogeneity-matrix interface. In contrast, for a pure circumferential eigenstrain distribution $\hat{\sigma}^{r r}$ and $\hat{\sigma}^{\theta \theta}$ are nonuniform in the inhomogeneity, with $\hat{\sigma}^{r r}$ being continuous at the inhomogeneitymatrix interface.

\section{Acknowledgement}

This research was partially supported by AFOSR - Grant No. FA9550-12-1-0290 and NSF - Grant No. CMMI 1130856. SS was supported by a Fulbright Grant.

\section{References}

[1] L. Tao, K. Rajagopal, On an inhomogeneous deformation of an isotropic compressible elastic material, Archives of Mechanics 42 (6) (1990) 729-736.

[2] D. Fu, K. Rajagopal, A. Szeri, Non-homogeneous deformations in a wedge of Mooney-Rivlin material, International Journal of Non-Linear Mechanics 25 (4) (1990) 375-387.

[3] K. Rajagopal, M. Carroll, Inhomogeneous deformations of non-linearly elastic wedges, International journal of solids and structures 29 (6) (1992) 735-744.

[4] K. Rajagopal, L. Tao, On an inhomogeneous deformation of a generalized neo-Hookean material, Journal of elasticity 28 (2) (1992) 165-184.

[5] J. R. Walton, J. P. Wilber, Deformations of Neo-Hookean Elastic Wedge Revisited, Mathematics and Mechanics of Solids 9 (3) (2004) 307-327.
[6] J. Walton, Vibrations of neo-Hookean elastic wedge, International journal of non-linear mechanics 38 (9) (2003) 12851296.

[7] T. Mura, Micromechanics of Defects in Solids, Martinus Nijhoff, 1982.

[8] K. Kondo, A Proposal of a New Theory concerning the Yielding of Materials based on Riemannian Geometry, The Journal of the Japan Society of Aeronautical Engineering 2 (8) (1949) 29-31, ISSN 1883-549X.

[9] Y. Ueda, K. Fukuda, K. Nakacho, S. Endo, A New Measuring Method of Residual Stresses with the Aid of Finite Element Method and Reliability of Estimated Values, Transactions of JWRI 4 (2) (1975) 123-131, ISSN 03874508.

[10] J. D. Eshelby, The determination of the elastic field of an ellipsoidal inclusion, and related problems, in: Proceedings of the Royal Society of London A: Mathematical, Physical and Engineering Sciences, vol. 241, The Royal Society, 376-396, 1957.

[11] T.-S. Jun, A. M. Korsunsky, Evaluation of residual stresses and strains using the eigenstrain reconstruction method, International Journal of Solids and Structures 47 (13) (2010) 1678-1686.

[12] C. Kim, P. Schiavone, A circular inhomogeneity subjected to non-uniform remote loading in finite plane elastostatics, International Journal of Non-Linear Mechanics 42 (8) (2007) 989-999.

[13] C. Kim, M. Vasudevan, P. Schiavone, Eshelby's conjecture in finite plane elastostatics, The Quarterly Journal of Mechanics and Applied Mathematics 61 (1) (2008) 63-73.

[14] C. Kim, P. Schiavone, Designing an inhomogeneity with uniform interior stress in finite plane elastostatics, Acta Mechanica 197 (3-4) (2008) 285-299.

[15] C.-Q. Ru, P. Schiavone, On the elliptic inclusion in anti-plane shear, Mathematics and Mechanics of Solids 1 (3) (1996) 327-333.

[16] C. Ru, P. Schiavone, L. Sudak, A. Mioduchowski, Uniformity of stresses inside an elliptic inclusion in finite plane elastostatics, International Journal of Non-Linear Mechanics 40 (2) (2005) 281-287.

[17] S. S. Antman, M. M. Shvartsman, The shrink-fit problem for aeolotropic nonlinearly elastic bodies, Journal of elasticity 37 (2) (1994) 157-166.

[18] A. Yavari, A. Goriely, Nonlinear elastic inclusions in isotropic solids, Proceedings of the Royal Society A: Mathematical, Physical and Engineering Science 469 (2160) (2013) 20130415

[19] A. Yavari, A. Goriely, The twist-fit problem: finite torsional and shear eigenstrains in nonlinear elastic solids, Proceedings of the Royal Society of London A: Mathematical, Physical and Engineering Sciences 471 (2183), ISSN 1364-5021.

[20] J. Marsden, T. Hughes, Mathematical Foundations of Elasticity, Dover Civil and Mechanical Engineering Series, Dover, ISBN 9780486678658, 1983.

[21] A. Yavari, A. Goriely, Riemann-Cartan geometry of nonlinear dislocation mechanics, Archive for Rational Mechanics and Analysis 205 (1) (2012) 59-118.

[22] T. Doyle, J. Ericksen, Nonlinear elasticity, Advances in applied mechanics 4 (1956) 53-115.

[23] A. Ozakin, A. Yavari, A geometric theory of thermal stresses, Journal of Mathematical Physics 51 (2010) 032902.

[24] A. Yavari, A geometric theory of growth mechanics, Journal of Nonlinear Science 20 (6) (2010) 781-830.

[25] A. Yavari, A. Goriely, Weyl geometry and the nonlinear mechanics of distributed point defects, Proceedings of the Royal Society A: Mathematical, Physical and Engineering Science 468 (2148) (2012) 3902-3922.

[26] A. Yavari, A. Goriely, The geometry of discombinations and its applications to semi-inverse problems in anelasticity, Proceedings of the Royal Society A: Mathematical, Physical and Engineering Science 470 (2169) (2014) 20140403.

[27] A. Yavari, A. Goriely, On the stress singularities generated by anisotropic eigenstrains and the hydrostatic stress due to annular inhomogeneities, Journal of the Mechanics and Physics 
of Solids 76 (2015) 325-337.

[28] S. Sadik, A. Yavari, Geometric nonlinear thermoelasticity and the time evolution of thermal stresses, Mathematics and Mechanics of Solids (2015) 1081286515599458.

[29] S. Sadik, A. Angoshtari, A. Goriely, A. Yavari, A Geometric
Theory of Nonlinear Morphoelastic Shells, Under review .

[30] C. Truesdell, The Physical components of vectors and Tensors, ZAMM-Journal of Applied Mathematics and Mechanics/Zeitschrift für Angewandte Mathematik und Mechanik $33(10-11)(1953) 345-356$. 